\title{
Point mutations affecting yeast prion propagation change the structure of its amyloid fibrils
}

Anna I. Sulatskaya ${ }^{1, \dagger}$, Stanislav A. Bondarev ${ }^{2,3, \dagger}$, Maksim I. Sulatsky ${ }^{4}$, Nina P. Trubitsina ${ }^{2}$, Mikhail V. Belousov ${ }^{2,5}$, Galina A. Zhouravleva, ${ }^{2,3}$, Manuel A. Llanos ${ }^{6}$, Andrey V. Kajava ${ }^{7,8}$, Irina M. Kuznetsova ${ }^{1}$, Konstantin K. Turoverov ${ }^{1, *}$

${ }^{1}$ Laboratory for Structural Dynamics, Stability and Folding of Proteins, Institute of Cytology Russian Academy of Sciences, Saint Petersburg, Tikhoretsky Ave. 4, 194064, Russia

${ }^{2}$ Department of Genetics and Biotechnology, Saint Petersburg State University, Saint Petersburg, Universitetskaya Emb. 7-9, 199034, Russia

${ }^{3}$ Laboratory of Amyloid Biology, Saint Petersburg State University, Saint Petersburg, 199034, Russia

${ }^{4}$ Laboratory of Cell Morphology, Institute of Cytology Russian Academy of Sciences, Saint Petersburg, Tikhoretsky Ave. 4, 194064, Russia

${ }^{5}$ Laboratory for Proteomics of Supra-Organismal Systems, All-Russia Research Institute for Agricultural Microbiology, Pushkin, Saint Petersburg, Podbelskogo Hwy. 3, 196608, Russia

${ }^{6}$ Laboratory of Bioactive Research and Development (LIDeB), CONICET, Department of Biological Sciences, Faculty of Exact Sciences, National University of La Plata, Buenos Aires, Argentina

${ }^{7}$ CRBM, CNRS, France; Université Montpellier, Cedex 5, France

${ }^{8}$ Institute de Biologie Computationnelle, Montpellier, Cedex 5, France

${ }^{\dagger}$ These authors contributed equally to the work

*Corresponding author: K.K. Turoverov, e-mail: kkt@incras.ru

Running title: Point mutations change the structure of prion amyloid fibril

Keywords: amyloid fibril, [PSI+] prion, Sup35p, point mutation, structural polymorphism, betaserpentine, super-pleated beta-structure, equilibrium microdialysis, thioflavin $\mathrm{T}$, binding stoichiometry 


\begin{abstract}
We investigated the effect of the point substitutions in the N-terminal domain of the yeast prion protein Sup35 (Sup35NMp) on the structure of its amyloid fibrils. As the objects of the study, proteins with mutations that have different influence on the $\left[\mathrm{PSI}^{\dagger}\right]$ prion propagation, but do not prevent the aggregation of Sup35NMp in vitro were chosen. The use of the wide range of physico-chemical methods allowed us to show significant differences in the structure of these aggregates, their physical size, clumping tendency. Also we demonstrated that the fluorescent probe thioflavin $\mathrm{T}$ can be successfully used for investigation of subtle changes in the structural organization of fibrils formed from various Sup35NMp. The obtained results and our theoretical predictions allowed us to conclude that some of selected amino acid substitutions delimit the region of the protein that forms the core of amyloid fibrils, and change the fibrils structure. The relationship of structural features of Sup35NMp amyloid aggregates, prepared in vitro, with the stability of the $\left[P S I^{+}\right]$prion in vivo allowed us to suggest that oligopeptide repeats $(\mathrm{R})$ of the amyloidogenic N-terminal domain of Sup35NMp from R0 to R2 play a key role in protein aggregation. Their arrangement rather than just presence is critical for propagation of the strong $\left[\mathrm{PSI}^{+}\right]$prion variants. The results confirm the suitability of the proposed combination of theoretical and empirical approaches for identifying changes in the amyloid fibrils structure, which, in turn, can significantly affect both the functional stability of amyloid fibrils and their pathogenicity.
\end{abstract}




\section{Introduction}

Amyloid fibrils are formed due to intermolecular interactions of the proteins. Their accumulation in the human organism accompanies many serious diseases, such as Alzheimer's and Parkinson's diseases, prion diseases, diabetes, etc. (1-7) In addition, an increasing number of studies indicate that fibrils can be not only a pathogenic factor, but also perform vital functions, and their implementation is probably closely related to the structural features of these protein aggregates (8-11). Despite the great diversity of the sequences and size of amyloidogenic regions in proteins, their amyloid fibrils have some common features. All of them are long thin unbranched protein aggregates enriched by $\beta$-sheets (12-14). The study of the structural organization of amyloids at the atomic level is hampered by the impossibility of applying conventional methods of X-ray crystallography, since amyloid fibrils cannot be crystallized. However, recently, a number of new methods (solid state NMR and Cryo-electron microscopy) were developed to study these protein aggregates (15). As a result, it was shown that the cross- $\beta$ amyloid fibrils formed from various amyloidogenic proteins have different structural variants $(16,17)$. This is also true for fibrils formed from the same protein under different conditions of fibrillogenesis or from a protein with altered amino acid sequence (18-23). The relevance of the study of amyloid polymorphism increased in the last decade due to the discovered association of the amyloid fibrils structure with their cytotoxicity, as well as with the variability of amyloidosis (18,24-26).

One of the well-known amyloids is yeast Sup35 protein, aggregation of which leads to the appearance of the $\left[\mathrm{PSI}^{+}\right]$prion. In the cells with this prion, the Sup35 protein forms amyloid aggregates. The $\left[\mathrm{PSI}^{+}\right]$factor leads to nonsense suppression in vitro $(27)$ and in vivo $(28,29)$ and is probably an adaptive response of the cell to various stress effects (30-32). It was shown that $\left[\mathrm{PSI}^{+}\right]$has various variants (33). Their properties are associated with the structural polymorphism of Sup35p aggregates (34). In particular it was shown that amyloid fibrils of the [VH], [VK], and [VL] strains of $\left[P S I^{\dagger}\right]$ differ in their morphologies (35).

The N-terminal domain plays a major role in propagation of the $\left[\mathrm{PSI}^{+}\right]$prion, that was shown previously in vivo (36). This region forms the core of amyloid fibrils (37-39) and its general structural arrangement can be represented well by the superpleated $\beta$-structure with parallel and in register stacking of $\beta$-strands (40-42). Sup35p can form many different superpleated $\beta$-structures that differ in their properties (40). Recently, we obtained a set of mutant forms of the Sup35p fragment (Sup35NMp) (reviewed in the work (43)). Each of these mutant forms has two glutamine residues (or one glutamine residue in combination with another amino acid) replaced with positively charged lysine residues in one of the six oligopeptide repeats of the N-domain (Table 1) (44). Based on the above-mentioned model of the superpleated $\beta$-structure (40) the assumption was made that if such mutations are located within the amyloid core this should result in a destabilization of prion aggregates. According to the experimental results, only three of the six alleles (sup35-M0 
(Q33K/A34K); sup35-M1 (Y46K/Q47K); sup35-M2 (Q61K/Q62K)), affect the stability and properties of $\left[P S I^{+}\right]$, both in combination with the wild-type allele and in the homozygous state. The remaining three alleles (sup35-M3 (Q70K/Q71K); sup35-M4 (Q80K/Q81K); sup35-M5 (Q89K/Q90K)) do not change (sup35-M3) or only slightly change the properties of $\left[\mathrm{PSI}^{+}\right](44-46)$. We hypothesized that these mutations affect the properties of the $\left[\mathrm{PSI}^{+}\right]$prion by changing the structure of Sup35NMp aggregates. The aim of this work was to study the structural polymorphism of amyloid fibrils formed from mutant forms of Sup35NMp using multidisciplinary approaches, as well as analyzing the relationship of the structural features of Sup35NMp amyloid aggregates obtained in vitro, with stability of the prion $\left[\mathrm{PSI}^{+}\right]$in vivo. For this purpose, we used conventional methods of structural analysis of amyloids such as electron microscopy and different spectroscopic approaches, but also a new approach based on the quantification of the binding of ThT fluorescent probe to the amyloid samples.

\section{Results}

Physico-chemical properties of amyloid fibrils formed from Sup35NMp and its mutant

\section{forms}

For the morphological characteristics of amyloid fibrils formed from Sup35NMp and its mutant forms, first of all, electron microscopy was used (Fig. 1). The obtained images allowed us to conclude that the mutant forms of Sup35NMp, like the wild-type protein, form unbranched amyloid fibrils of various morphologies in vitro. In particular, it was shown that wild-type Sup35NMp fibrils (Fig. 1A) have the greatest length (>500 nm) and diameter (up to $20 \mathrm{~nm}$ ), as well as are most clumped aggregates compared to fibrils formed from some mutant forms of this protein. Substitutions Q33K/A34K at the beginning of the N-terminal domain noticeably decrease the length (up to $200 \mathrm{~nm}$ ) and thickness (about $10 \mathrm{~nm}$ ) of amyloid fibrils (Fig. 1B). When replacing Y46K/Q47K and Q61K/Q62K, fibrillar structures of various lengths can be observed in the sample: from 40 to 200 (Fig. 1C) and from 100 to $350 \mathrm{~nm}$ (Fig. 1D), respectively. At the same time the replacement Q70K/Q71K leads to the formation of predominantly long fibrils (up to $500 \mathrm{~nm}$ ) (Fig. 1E). Substitutions Q80K/Q81K and Q89K/Q90K in the last two oligopeptide repeats of Sup35NMp lead to the formation of long amyloid fibrils with the greatest diameter and clumping tendency (Fig. 1F,G), compared with fibrils formed form remaining mutant protein forms. It can be noted that the replacement Q89K/Q90K has the least impact on the physical size and clustering of Sup35NMp amyloid fibrils (Fig. 1G). Images obtained by electron microscopy for this sample and wild-type Sup35NMp amyloid fibrils are almost identical (Fig. 1A,G). Thus, the obtained results allowed us to conclude that there are significant differences in the overall morphology of amyloid fibrils formed from Sup35NMp with point mutations in its amino acid sequence. 
Prepared amyloid fibrils were also studied using a number of spectroscopic approaches. The absorption spectra of Sup35NMp amyloid fibrils, as expected, have a maximum at a wavelength $\lambda_{\max }$ $=276 \mathrm{~nm}$ (tyrosine absorption maximum), since there are no tryptophan residues in the amino acid sequence of the studied proteins (Fig. 2A). Analysis of the absorption spectra of amyloid fibrils was carried out taking into account their light scattering by the standard procedure (47). It should be noted that the turbidity of the samples within the error does not change (Fig. 2C), despite the different size of amyloid fibrils. This fact may be related to the different concentrations of the fibrils. The observed difference in fibrils length (Fig. 1) in the samples with identical protein concentration and similar rate of the monomeric proteins aggregation (supplementary Fig. S1A) confirmed such differences. Thus, it can be assumed that the size of the Sup35NMp aggregates is inversely proportional to their number, which causes the same turbidity of the samples and does not contradict with the electron microscopy data (Fig. 1). The fluorescence spectra of Sup35NMp amyloid fibrils have a maximum at a wavelength of $\sim 305 \mathrm{~nm}$, which corresponds to the tyrosine fluorescence wavelength (Fig. 2D). At the same time the fluorescence spectra of some types of amyloid fibrils have a shoulder in the longwavelength region, which may indicate the formation of tyrosinate, a special form of tyrosine, the formation of which is caused by the microenvironment of this amino acid residue. This assumption is confirmed by the fact that the amino acid substitutions in Sup35NMp are localized in a close proximity to several tyrosine residues (Table 1), which means that amino acid replacement can lead to a change in the microenvironment of these residues. To determine the fluorescence excitation spectra of the samples undistorted to the primary inner filter effect, a correction coefficient was used, which is determined only by the total optical density of the solution (48). The corrected excitation spectra (Fig. 2B), as expected, almost coincide with the absorption spectra of the studied solutions, which indicate the homogeneity (identity) of the absorbing centers in the samples.

To estimate the secondary structure of Sup35NMp amyloid fibrils we measured the CD spectra of samples in the far-UV region (in the wavelength range 200-260 nm) (Fig. 2E). The recorded CD spectra were analyzed by the special software K2D2 and CDPro (using three different regression methods of Selcon, Contin, and CDSSTR) and several basic sets of proteins with a known secondary structure (the sets contain from 37 to 56 proteins with different structure, including soluble, membrane and denatured). The results indicate that despite the obvious differences in the shape of the CD spectra of amyloid fibrils formed from various Sup35NMp forms, the content of the secondary structure in the samples differs slightly (in particular, the content of $\beta$-sheets is about $35 \pm 3 \%$ ). This may be due to the fact that the standard basic sets of proteins used for the calculation are not representative for the analysis of the spectra of protein aggregates. Since the quantitative analysis of the CD spectra of fibrils is rather arbitrary, we only performed a visual analysis of the registered CD spectra. For this aim, CD spectra of proteins and peptides with representative secondary structures were used (49). Fibrils formed from the wild-type Sup35NMp, as well as from the Sup35-M4p and 
Sup35-M5p, have apparent negative shoulder around $220 \mathrm{~nm}$, that allows us to suggest the essential content of ordered $\beta$-structure. At the same time in the case of the fibrils formed from Sup35-M0p Sup35-M3p this shoulder becomes significantly smaller (Fig. 2F), and the shoulder around $200 \mathrm{~nm}$ that is typical for unfolded proteins (50) becomes predominant. In conclusion, it can be noted that according to the published data (see, for example, work by Tinoco et al. (51)), light scattering of macromolecules can substantially distort the CD spectra. However, since the turbidity of the studied samples is the same (Fig. 2C), we suggested that even if their CD spectra are distorted, this occurs to the same extent for all samples. In this regard, on the basis of the obtained data, we can reliably confirm that the CD spectra of the samples of amyloids formed from Sup35NMp with modified amino acid sequence vary significantly, and therefore the structure of the fibrils is also different. Observed differences in the content of the $\beta$-folded structure allowed us to suggest the differences in the length of the amino acid sequence forming the backbone of amyloid fibrils.

\section{Interaction of Sup35NMp amyloid fibrils with a fluorescent probe thioflavin T}

The fluorescent dye thioflavin T (ThT) is a widely and effectively used probe to diagnose the formation of amyloid fibrils (52-54). Recent studies suggest that the dye can also be used to study the polymorphism of these protein aggregates. This is due to the fact that, according to modern concepts, ThT binding sites are located in the grooves along the long axis of the fibrils perpendicular to the $\beta$ sheets (55). The affinity and stoichiometry of the ThT interaction with the fibrils, as well as the photophysical characteristics of the dye bound to them, can vary significantly for different amyloid fibrils (56-60). This is a confirmation of amyloid polymorphism and can provide valuable and unique information about the features of their structure, which is difficult (or impossible) to obtain using other experimental approaches. In the present work we attempted to use this dye to assess structural differences of amyloid fibrils formed from various forms of Sup35NMp.

The analysis of ThT - amyloid fibrils interaction is associated with a serious methodological problem. The test samples always contain two fractions of the dye: free and bound to fibrils ThT. Moreover, to determine the characteristics of the ThT - amyloid fibrils interaction, it is necessary to separate the photophysical properties of these dye fractions. To solve this problem, we previously proposed a special approach based on the preparation of the tested solutions using equilibrium microdialysis (for details of the experiment, see our works $(22,61,62)$ ). In the present work using this approach we determined absorption spectra (Fig. 3C) and, therefore, concentrations of the free and bound to fibrils dye fractions in the prepared samples. This allowed us to plot the Scatchard dependencies characterizing the interaction of ThT with Sup35NMp amyloid fibrils, analysis of which allowed us to estimate the number of the dye binding modes to fibrils (Fig. 3A,B). The obtained results indicate the similarity of the dependencies for the Sup35-M4p and Sup35-M5p fibrils with the Scatchard plot for wild-type Sup35NMp fibrils, which may indicate the greatest similarity of these 
fibrils structure (this agrees well with the electron microscopy and CD spectroscopy data). The Scatchard dependencies for other samples differ significantly. The greatest difference can be noted for fibrils formed from Sup35-M1p and Sup35-M2p, in which the substitutions are located approximately in the middle of the amyloidogenic N-terminal domain of the protein. Intriguingly these substitutions lead to a decrease in stability and loss of the $\left[\mathrm{PSI}^{+}\right]$prion properties of the protein in vivo.

In order to investigate the mechanism of the impact of the substitutions made in the Sup35NMp amino acid sequence on the fibrils structure, we calculated stoichiometry and affinity of ThT interaction with amyloids (the method for determining these parameters is described in detail in the work (61)). Taking into account the obvious nonlinearity of the majority of the plotted Scatchard dependencies, experimental data were analyzed under the assumption of the existence of two different types of dye binding to fibrils (Table 2). In addition, fluorescence decay curves (Fig. 3F), fluorescence (Fig. 3D) and fluorescence excitation (Fig. 3E) spectra of samples prepared by equilibrium microdialysis were recorded. The fluorescence lifetime of the dye bound to amyloid fibrils formed from various Sup35NMp forms was determined (Fig. 3F (Inset)), and using specially developed protocols (61), the molar extinction coefficient and fluorescence quantum yield (Fig. 3D (Inset)) of ThT associated with different modes of the fibrils were also calculated (Table 2).

An analysis of the obtained results indicates a difference in the molar extinction coefficient, fluorescence quantum yield and lifetime of ThT bound to the studied amyloid fibrils. We previously showed that the increase in fluorescence quantum yield and lifetime of ThT when it binds to amyloid fibrils is due to the steric restriction of the dye fragments rotation relative to one another (causing nonradiative ThT deactivation) which in turn is determined by an increase of the rigidity of the dye microenvironment (63). In addition a change in the polarity of ThT microenvironment can result in an increase in its fluorescence quantum yield (64). The difference in the molar extinction coefficients of ThT bound to fibrils can also be due to the variety in the conformation of the dye, as well as the hydrophobicity and polarity of its microenvironment. All of the above means that the detected differences in the photophysical characteristics of ThT when the dye interacts with different variants of Sup35NMp fibrils may be due to differences in the side radicals that form the dye binding groove, and hence the amino acid sequence that forms the core of the fibrils.

Despite the shown differences in the microenvironment of the ThT molecules bound to fibrils the affinity of the dye interaction with different amyloids is almost the same. This suggests that the dye interacts with the same binding sites of tested amyloid fibrils with similar specificity. As noted above, the existence of one of the detected binding types is due to the incorporation of the dye into the grooves along the fiber axis (55). In our previous works, on the basis of the analysis of ThT binding parameters to a large number of different amyloid fibrils and their images obtained by electron microscopy, it was suggested that the second type of binding occurs during the clumping of amyloid fibrils. In this case the dye bound to the fibrils grooves located into an even more rigid 
microenvironment (compared to the first binding type). The calculated stoichiometry of the ThT interaction with the second binding mode and formation of large fibril clumps are related according to our observations (see Table 2 and Fig. 1). The largest number of ThT binding sites with this mode is shown in the case of fibrils formed from wild type protein and from the Sup35-M4p and Sup35-M5p, which, according to electron microscopy data, form the largest clumps. In addition, it is for these samples the largest shift of the absorption (Fig. 3C) and fluorescence excitation (Fig. 3E) spectra and increase of the fluorescence lifetime (Fig. 3F (inset)) of the bound ThT, as well as the maximum values of the fluorescence quantum yield of the dye associated with the second mode of the fibrils (Fig. 3D (Inset)) were observed. All this, according to the results of our previous works, is characteristic of amyloid fibrils prone to clumping and clustering (see, for example, our work (22)).

The number of ThT binding sites to the first mode of the amyloids should be determined by the number and length of the grooves formed by the amino acids of the fibril core (Fig. 4A). The number of binding grooves depends on the length of the fibrils, their number as well as the length of the amino acid sequence that forms the core of the fibrils. Given the fact that the protein concentration used for amyloid fibrils preparation and the ratio between aggregated and monomeric protein were the same, the length and number of amyloid fibrils should be interconnected (as evidenced by the same turbidity of the samples), but should not alter the number of binding grooves. This means that in the case of shorter fibrils, number of these aggregates should be greater (and vice versa). Although the electron microscopy cannot be used to clearly quantify the number of amyloid fibrils in a sample, it can be noted that the results used by this method do not contradict the made assumption. Thus, the main factor determining the number of the dye binding sites is probably the length of the amino acid sequence that forms the core of the amyloid fibrils, or more precisely the protofibrils. Thereby the different stoichiometry of ThT binding to Sup35NMp fibrils supported the initial hypothesis that the amino acid substitutions affect the length of the protein fragment involved in fibrillogenesis. Next, we modelled various parameters characterizing the structure of the fibrils formed from the mutant forms of Sup35NMp, using various in silico methods.

The relationship between thioflavin $T$ binding parameters to different Sup35NM aggregates and their structural arrangements

The investigated substitutions in Sup35NMp amino acid sequence were designed to affect the structure of this protein aggregates (44). The possible impact of these substitutions was analysed with the bioinformatic approaches. The ArchCandy program predicts amyloidogenic regions in the protein based on its ability to form specific structural motif ( $\beta$-strand - loop - $\beta$-strand), called $\beta$-arch (65). The accuracy of this tool was demonstrated in several works (65-67). According to the results of ArchCandy program introduced mutations change distributions of $\beta$-arches across the protein. The replacement of polar amino acid to charged one can lead to a noticeable decrease in the 
amyloidogenic potential of the corresponding region (Fig. 4B). The BetaSerpentine program, which allows reconstructing all possible $\beta$-serpentine arrangements for a given protein, was used to evaluate the impact of the mutations on the structure of Sup35 aggregates. Previously we demonstrated that the program predicts reliable structures, which correspond to the experimental data (68). The BetaSerpentine demonstrates that aggregates of Sup35NMp with investigated substitutions include protein fragments of different length (Fig. 4C). Thus the set of Sup35-M0-M5 proteins represents a good model for the investigation of changes in amyloid structure caused by point mutations. The analysis of ThT binding with aggregates may reveal corresponding structural rearrangements.

Two different models were proposed for the explanation of ThT binding to amyloids fibrils. One of them, which was mentioned above, assumes that the dye is incorporated into grooves formed by the side chains of amino acids, located in $\beta$-strands (Fig. 4A, $\beta$-sheet groove or b-groove model) (55). This mode of ThT-binding was confirmed by molecular dynamic simulations, which also reveal that the grooves with aromatic residues possess a higher affinity to the ThT $(56,57)$ (for review see work by Biancalana \& Koide (58)). This model was experimentally proved for $\beta$-sheet with antiparallel $\beta$-strands. In such arrangement minimal ThT binding site is formed by four $\beta$-strands and optimal - by five $(56,57)$. In the other model, the ThT binds to the groove formed by the side chains and backbone at the base of the $\beta$-arch (arch groove or a-groove model) (40). First of all, this model was proposed to explain the interaction of the Congo Red with Ure2p fibrils. This a-groove model can be also applied to the ThT molecule due to the similarity of two dyes (Fig. 4A). The results of molecular docking confirm that the ThT can bind with the comparable affinity to both types of the grooves (Fig. 4A). We demonstrated that four $\beta$-strands are required for the single ThT binding site for parallel super-pleated $\beta$-structure, which was proved for Sup35 aggregates $(41,42)$.

Using the BetaSerpentine tool (68) we can predict numbers of b-grooves and a-grooves $\left(\mathrm{N}_{\mathrm{b}}\right.$ grooves and $\mathrm{N}_{\mathrm{a} \text {-grooves }}$ ) which can provide sites for ThT binding according to different models (see Fig. S1 for an example), and then compare these theoretical values with experimental data. The program predicts hundreds of possible arrangements for the given protein (in case of Sup35NMp or its variants this number may exceed several thousands). In the further analysis, we used mean values for number of grooves or arches across all predicted structures. This parameter adequately describes the most probable characteristic of potential fibers reflecting the most frequent case among all possible variants (Fig. S2). We found significant correlation between the number of ThT binding sites for the second mode of amyloid fibrils formed from different proteins $\left(n_{2}\right.$ parameters, Table 2$)$ and the corresponding mean number of b- and a-grooves (Fig. 4D-E). We focused only on the second mode because it was proposed previously that it describes the incorporation of the dye along fibril, and the first mode is rather due to ThT binding to the fibril clumps (60). Following the idea that aromatic residues can increase the affinity of the groove to the dye, we checked the correlation between $n_{2}$ and mean numbers of b-grooves with one aromatic $(\mathrm{F}, \mathrm{W}, \mathrm{Y})$ and one aliphatic residue $(\mathrm{A}, \mathrm{L}, \mathrm{M}, \mathrm{I}, \mathrm{V}$; 
designated RL), grooves with one aromatic and any other residue (RA) and grooves with one hydrophobic and any other residue (F, W, Y, A, L, M, I, V; designated HA). The correlation was not observed only in the case of b-grooves of RL (Fig. 4D-E). Knowing that among thousands of possible arrangements of $\beta$-serpentines only several ones are present in the solution, we confirmed our analysis by the following procedure. We recalculated correlations for random sets of arrangements for each protein. One thousand independent samples were tested, and the significant correlation was found in most cases (Table S1).

The $n_{2}$ parameter corresponds to the number of ThT binding sites per protein molecule in the aggregate. According to the literature data and our calculations, at least $4 \beta$-strands (protein molecules) are required for the single ThT binding site in a single groove. Thus the simplest relationship between experimentally determined number of sites and predicted should be described by the equation $n_{2}=k * N$, where $N$ is a number of grooves, binding ThT, and $k$ is a number of protein molecules required for ThT binding in the single groove. According to our simulation $k$ should be equal to $1 / 4$. We made a regression analysis with our data and calculated corresponding coefficients (Fig. 4E). For the a-model the coefficient is in expected diapason, demonstrating a good match of experimental and theoretical data. For the b-model, the results significantly vary depending on the type of analyzed grove and the coefficients are close to expected only if we considered HA but not any or RA grooves. This is in agreement with previously published data about the role of aromatic and aliphatic residues in the ThT binding $(56,57)$. The major limitations of this analysis are the assumption that all ThT binding sites should be occupied and dissociation of the dye is negligible. Further simulations and experiments are required to propose a detailed model. These data can be used for the further improvement of the BetaSerpentine program. In general, theoretically predicted numbers of ThT binding sites correlate well with the experimentally determined ones. This result supports the reliability of BetaSerpentine predictions.

\section{Relationship of the structure of amyloid fibrils formed from various forms of Sup35NMp} obtained in vitro with the properties of the prion $\left[\mathrm{PSI}^{+}\right]$in vivo

The biophysical and bioinformatics analyses demonstrated structural differences between amyloid aggregates of investigated Sup35NM proteins. To reveal the relationship between amyloid structure and the prion properties we transformed yeast $\left[\right.$ psi $\left.^{-}\right]\left[\mathrm{pin}^{-}\right]$strain, bearing wild type allele of SUP35, with these aggregates and analyzed spectra of appeared $\left[P S I^{+}\right]$variants (Fig. 5). More than 600 obtained $\left[\mathrm{PSI}^{\dagger}\right]$ strains were analyzed among five thousand transformants selected. These $\left[\mathrm{PSI}^{+}\right]$ strains were differentiated into three groups: strong, weak and very weak, using OT56 and OT55 $(69,70)$ strains as references for strong and weak $\left[\mathrm{PSI}^{\dagger}\right]$ variants, respectively (Fig. 5). To exclude potential effects of the substitutions on the prion properties we used 2-OT56 yeast strain containing wild type $S U P 35$ allele. Thus, all newly obtained $\left[\mathrm{PSI}^{+}\right]$prion variants propagate in the presence of 
the same Sup35 protein. And all observed differences in the prion phenotype are linked to the specific structure of aggregates, but not to the protein sequence. In turn, structural differences between aggregate samples obtained in vitro and used for transformation are caused by the analyzed substitutions.

Fibrils of Sup35NM-M0, M1 and M2 led to the appearance of very weak prion. From the theoretical point of view amyloid core in these aggregates should be shorter compared to other investigated proteins. This hypothesis was supported at least by ThT binding data (smaller number of ThT binding sites for second mode, Table 2) and by BetaSerpentine analysis (Fig. 4C). In opposite case, the aggregates of Sup35NM or Sup35NM-M3,-M4 and -M5 possessing longer amyloid core led to the formation of strong prion variants with higher frequency. This observation is in disagreement with the previous work demonstrating that short amyloid core is a characteristic of the strong $\left[\mathrm{PSI}^{+}\right]$ variants $(38,39)$. At the same time, our results supported a critical role of QN-region and first two oligopeptide repeat in the prion propagation (71-73). Recently it was shown that Sup35 region 42-72 is more resistant to the proteinase $\mathrm{K}$ digestion in the strong prion variants (74). The mutations sup35$M 1$ and $-M 2$ lead to substitutions inside this region. Thus our results supported the idea that first two oligopeptide repeats should be included into amyloid core for the appearance of strong prion variant. We also can conclude that the length of Sup35 amyloid core is not a key parameter for the $\left[\mathrm{PSI}^{+}\right]$ prion properties determination. We need to mention that the highest rate of strong prion variants corresponded to Sup35NM-M4 fibrils. This is only observation and this fact have not statistical support, but it is in good agreement with our results demonstrated that sup35-M4 change the prion variant to a stronger one (44). The role of the first two Sup35 oligopeptide repeats in the prion maintenance was shown previously. The deletion of these regions abolishes $[\mathrm{PSI}+]$ propagation, but duplication increases its de novo formation $(72,73,76)$. Our results together with data published by Dergalev et al., 2019 suggest that propagation of the strong prion variants requires not only the presence of the two first oligopeptide repeats but also their implication into the fibril structure.

According to our previous results, three of the six alleles (sup35-M0, sup35-M1 и sup35-M2) affect stability of $\left[\mathrm{PSI}^{\dagger}\right]$. At the same time the remaining three alleles (sup35-M3, sup35-M4 и sup35M5) are comparable or only slightly change the properties of $\left[\mathrm{PS}^{+}\right](44,46)$. Using electron microscopy, we found differences in the fibril structure of the Sup35NMp with the amino acids substitutions. Despite that, several investigated mutations (sup35-M0, sup35-M1 and sup35-M2) destabilize the prion, our results revealed that corresponding proteins are able to form aggregates. This fact is in agreement with published data describing variant specificity of sup 35 mutations. They may destabilize one prion variant but be compatible with another $(10,66,75)$. It is likely that effect of a certain mutation on the prion depend on the structural variants of the corresponding Sup35p aggregates. 


\section{Conclusion}

In the present work, the effect of point amino acid replacements in the fragment of the yeast prion protein Sup35 (Sup35NM) on the structural organization of its amyloid fibrils was investigated. Mutant proteins containing amino acid substitutions in one of the six oligopeptide repeats of the Sup35NMp amyloidogenic N-domain were chosen as objects of the study. The choice of positions for mutations was based on the model of the superpleated $\beta$-structure of Sup35NMp amyloid fibrils. It was shown that despite the fact that the made substitutions do not prevent the formation of amyloid fibrils of this protein, they have a significant impact on the different aggregate properties.

One of the main experimental approaches that made it possible to conduct a comparative study of the structural organization of Sup35NMp amyloid fibrils was investigating the interaction of a specific probe thioflavin $\mathrm{T}$ (ThT) with the fibrils using equilibrium microdialysis. It was shown that substitutions in the Sup35NMp N-domain significantly affect the stoichiometry of the dye-fibrils interaction, as well as the photophysical characteristics of the bound ThT (in particular, its fluorescence quantum yield and lifetime). This, in turn, indicates a significant difference in the structural organization of the fibrils sites with which ThT binds, and hence the difference in the structure of amyloid fibrils formed from various mutant Sup35NMp forms. An analysis of the stoichiometry of ThT interaction with different variants of Sup35NMp fibrils allowed us to make a suggestion that the amino acid substitutions affect the length of the protein fragment involved in fibrillogenesis.

Prediction of structural arrangements of amyloid fibrils by Arch Candy is in line with our hypothesis that the investigated amino acid substitutions in Sup35p reduce the length of the protein fragment(s) involved in the formation of the amyloid fibril core. Moreover, the BetaSerpentine program agrees well with the experimental data about interaction of ThT with Sup35p aggregates. We showed a good correlation of the experimentally calculated number of ThT binding sites of Sup35NMp fibrils per protein molecule with expected numbers of binding sites (b- or a-grooves), calculated from structural prediction. The obtained results are in a good agreement with the existing models of ThT - amyloid fibrils interaction (b-groove and a-groove models). Finally, our results supported the importance of amyloid conformation of the first two oligopeptide repeats for the propagation of the $\left[\mathrm{PSI}^{+}\right]$prion.

The results confirm the sensitivity of the combined theoretical and empirical approach to detecting minor changes in the structure of amyloid fibrils (for example, caused by various external factors or point mutations) that are difficult to detect using other techniques. Obtained results can be used in the study of polymorphism and the mechanisms of formation of amyloid fibrils (including amyloids formed from prion proteins), as well as in the development of approaches to the diagnosis and treatment of amyloidosis. 


\section{Experimental procedures}

\section{Materials}

Fluorescent dyes Thioflavin T (ThT) "UltraPure Grade" (AnaSpec, USA) and ATTO-425 (ATTO-TEC, Germany) were used without after-purification for mature amyloid fibrils study.

\section{Protein purification and aggregation}

For Sup35NMp purification, pET20b-SUP35NM (77) plasmid or its derivatives obtained by side-directed mutagenesis (primer sequences are available upon request) were used. For protein purification, E.coli strain BL21(DE3) was used (78). Overproduction of recombinant proteins was carried out in 2TYa media with $1 \mathrm{mM}$ IPTG. Cultures were grown at $37^{\circ} \mathrm{C}$ for 6 hours. Proteins were purified in denaturing conditions (in presence of $8 \mathrm{M}$ urea) according to previously published protocols (79). The purification was performed with a two-step purification procedure with Ni-NTA agarose (Invitrogen) and Q-sepharose (GE Healthcare) columns. Proteins were concentrated with a centricon $30 \mathrm{kDa}$ (Millipore). For aggregation Sup35NM proteins were diluted at least 100-fold into fibril assembly buffer $(5 \mathrm{mM}$ potassium phosphate $\mathrm{pH} 7.5,150 \mathrm{mM} \mathrm{NaCl})$ to the final concentration $0.5 \mathrm{mg} / \mathrm{ml}$. The protein solution was incubated at $25^{\circ} \mathrm{C}$ with overhead rotation (approximately 60 times per minute). To monitor amyloid fibril formation, aliquots were removed every 12 hours up to 24 hours of incubation. The rate of aggregated protein was estimated by SDS-PAGE with boiled and unboiled samples. The aggregation was stopped when rate of the monomeric protein was below $30 \%$.

\section{Protein transformation}

Protein transformation was performed based on previously published protocol $(80,81)$ and [psi $]$ 2-OT56 yeast strains was used (82). To prepare spheroplasts for transformation yeast cells were grown in YPD media to OD600 $=0.3$ and successively washed with sterile $\mathrm{H}_{2} \mathrm{O}$, buffer $(1 \mathrm{M}$ sorbitol, $25 \mathrm{mM}$ EDTA, $50 \mathrm{mM}$ dithiothreitol), $1 \mathrm{M}$ sorbitol, and SCE buffer (1 M sorbitol, $1 \mathrm{mM}$ EDTA, 10 $\mathrm{mM}$ sodium citrate, $\mathrm{pH} 5.8)$. Cells were spheroplasted with $50 \mu 1$ of zymolyase $(120 \mathrm{U} / \mathrm{ml})$ in SCE buffer at $30^{\circ} \mathrm{C}$ for $30 \mathrm{~min}$. Spheroplasts were successively washed with $1 \mathrm{M}$ sorbitol and STC buffer (1 M sorbitol, $10 \mathrm{mM} \mathrm{CaCl}_{2}, 10 \mathrm{mM}$ Tris, $\mathrm{pH}$ 7.5). Pelleted cells were resuspended in $1 \mathrm{ml}$ of STC buffer. A $100 \mu 1$ portion of the spheroplast suspension was mixed with $2 \mu 1$ of salmon sperm DNA (5 $\mathrm{mg} / \mathrm{ml}$ ), $500 \mathrm{ng}$ of selectable plasmid (pRS316) (83) and $5 \mu 1$ of Sup35NM filaments in concentration $0.5 \mu \mathrm{g} / \mathrm{ml}$. The mixture was incubated during $30 \mathrm{~min}$ at room temperature. Fusion was induced by addition of $900 \mu \mathrm{l}$ of PEG buffer (20\% (w/v) PEG 8000, $10 \mathrm{mM} \mathrm{CaCl}_{2}, 10 \mathrm{mM}$ Tris, $\mathrm{pH}$ 7.5) for 30 min. Cells were centrifuged, resuspended in $150 \mu \mathrm{l}$ of SOS buffer ( $1 \mathrm{M}$ sorbitol, $7 \mathrm{mM} \mathrm{CaCl}_{2}, 0.25 \%$ yeast extract, $0.5 \%$ bacto-peptone), incubated at $30^{\circ} \mathrm{C}$ for $30 \mathrm{~min}$ and plated on synthetic media (SC) (84) with $1 \mathrm{M}$ sorbitol lacking uracil. Then transformants were plated on the $1 / 4$ YEPD media (85) to determine presence of the prion and classify the $\left[P S I^{+}\right]$variants. The OT55 and OT56 $(69,70)$ were used as a "standard" weak and strong variants respectively. 


\section{Spectroscopic studies}

The absorption spectra of the samples were recorded using a U-3900H spectrophotometer (Hitachi, Japan). The absorption spectra of amyloid fibrils and ThT in the presence of the fibrils were analyzed along with the light scattering using a standard procedure (47). The concentrations of ThT and Sup3NMp in solution were determined using molar extinction coefficients of $\varepsilon_{412}=31600 \mathrm{M}^{-1} \mathrm{~cm}^{-}$ ${ }^{1}$ and $\varepsilon_{276}=29000 \mathrm{M}^{-1} \mathrm{~cm}^{-1}(86)$, respectively.

Fluorescence and fluorescence excitation spectra were measured using a Cary Eclipse spectrofluorimeter (Varian, Australia). Florescence of the Sup35NMp fibrils was excited at $280 \mathrm{~nm}$ and registered at $305 \mathrm{~nm}$. Fluorescence of ThT and fluorescent dye ATTO-425 was excited at a wavelength of $440 \mathrm{~nm}$. A PBS solution of ATTO-425, whose fluorescence and absorption spectra are similar to that of ThT, was taken as a reference for determining the fluorescence quantum yield of ThT bound to fibrils. The fluorescence quantum yield of ATTO- 425 was taken as 0.9 (ATTO-TEC Catalogue 2009/2010 p.14). The spectral slits width was $5 \mathrm{~nm}$ in most of experiments. Changing the slit widths did not influence the experimental results. Recorded fluorescence intensity was corrected on the primary inner filter effect with the use of previously elaborated(48) and successfully applied for ThT (87) approach.

CD spectra in the far UV-region were measured using a J-810 spectropolarimeter (Jasco, Japan). Spectra were recorded in a $0.1 \mathrm{~cm}$ cell from 260 to $200 \mathrm{~nm}$. For all spectra, an average of three scans was obtained. The CD spectrum of the appropriate buffer was recorded and subtracted from the samples spectra. Amyloid fibrils in concentration $\sim 0.2 \mathrm{mg} / \mathrm{ml}$ were used.

Fluorescence decay curves were recorded by a spectrometer FluoTime 300 (PicoQuant, Germany) with the Laser Diode Head LDH-C-440 $\left(\lambda_{e x}=440 \mathrm{~nm}\right)$. The fluorescence of ThT was registered at $\lambda_{e m}=490 \mathrm{~nm}$. ThT in concentration $\sim 2.2 \cdot 10^{-5} \mathrm{M}$ and amyloid fibrils in concentration $0.2 \mathrm{mg} / \mathrm{ml}$ were used. The measured emission decays were fit to a multiexponential function using the standard convolute-and-compare nonlinear least-squares procedure (88). In this method, the convolution of the model exponential function with the instrument response function (IRF) was compared to the experimental data until a satisfactory fit was obtained. The fitting routine was based on the nonlinear least-squares method. Minimization was performed according to Marquardt (89).

\section{Electron microscopy}

Micrographs were obtained using a transmission electron microscope Libra 120 (Carl Zeiss, Germany). Amyloid fibrils were placed on nickel grids coated with formvar films (Electron Microscopy Sciences, USA). To obtain electron micrographs, the method of negative staining with a $1 \%$ aqueous solution of uranyl acetate was used.

\section{Preparation of the samples for the study of ThT- amyloid fibrils interaction}

ThT-fibrils tested solutions were prepared by equilibrium microdialysis using a Harvard Apparatus/Amika (USA) device in the same environment in which fibrils were obtained. The stability 
of the structure and properties of amyloid fibrils during the equilibrium microdialysis experiment was experimentally proven. Equilibrium microdialysis was performed for a fixed concentration of fibrils $(\sim 0.4 \mathrm{mg} / \mathrm{ml})$ and a wide range of concentrations of ThT $(2-140 \mu \mathrm{M})$. Further spectroscopic study of these sample and reference solutions allowed calculating ThT-fibrils affinity and stoichiometry. Details of experimental procedure see in the Supporting information.

\section{Computational approaches}

The ArchCandy program with recommended parameters (65) was used for the prediction of amyloidogenic properties of proteins. The predictions of $\beta$-serpentine arrangements were performed with the BetaSerpentine program (68).

In order to identify putative binding sites for ThT, a blind docking experiment was conducted. Whereas in conventional docking simulations a ligand is docked into a small portion of the protein (binding pocket), blind docking refers to docking a ligand to the whole surface of a protein without any prior knowledge of the target binding site. By clustering the obtained poses and analyzing their interaction energies, putative binding pockets for a probe molecule can be identified. Thioflavin $\mathrm{T}$ was docked into the typical $\beta$-arcade structure (PDB id: 2BEG) using the Achilles Blind Docking Web Server (90) available at: http://bio-hpc.ucam.edu/aquiles/.

\section{Acknowledgments}

This work was supported by RF President Fellowship (SP-841.2018.4, polymorphism of the fibrils); grant from Russian Foundation for Basic Research (№ 17-54-150002), Research Resource Center for Molecular and Cell Technologies SPbSU and PRC CNRS grant PRC1524 (relationship between ThT binding parameters and their structural arrangements).

Conflict of interest: The authors declare that they have no conflicts of interest with the contents of this article.

\section{References}

1. Harper, J. D., Lieber, C. M., and Lansbury, P. T., Jr. (1997) Atomic force microscopic imaging of seeded fibril formation and fibril branching by the Alzheimer's disease amyloid-beta protein. Chem. Biol. 4, 951-959

2. Kelly, J. W. (1997) Amyloid fibril formation and protein misassembly: a structural quest for insights into amyloid and prion diseases. Structure 5, 595-600

3. Carrell, R. W., and Gooptu, B. (1998) Conformational changes and disease-serpins, prions and Alzheimer's. Curr. Opin. Struct. Biol. 8, 799-809

4. Hashimoto, M., and Masliah, E. (1999) Alpha-synuclein in Lewy body disease and Alzheimer's disease. Brain Pathol. 9, 707-720 
5. Koo, E. H., Lansbury, P. T., Jr., and Kelly, J. W. (1999) Amyloid diseases: abnormal protein aggregation in neurodegeneration. Proc. Natl. Acad. Sci. U S A 96, 9989-9990

6. Uversky, V. N., Talapatra, A., Gillespie, J. R., and Fink, A. L. (1999) Protein deposits as the molecular basis of amyloidosis. Part I. Systemic amyloidoses. Med. Sci. Monitor 5, 1001-1012

7. Uversky, V. N., Talapatra, A., Gillespie, J. R., and Fink, A. L. (1999) Protein deposits as the molecular basis of amyloidosis. II. Localized amyloidosis and neurodegenerative disorders. Med. Sci. Monitor 5, 1238-1254

8. Patino, M. M., Liu, J.-J., Glover, J. R., and Lindquist, S. (1996) Support for the prion hypothesis for inheritance of a phenotypic trait in yeast. Science 273, 622-626

9. Paushkin, S., Kushnirov, V., Smirnov, V., and Ter-Avanesyan, M. (1996) Propagation of the yeast prion-like $\left[\mathrm{PSI}^{+}\right]$determinant is mediated by oligomerization of the SUP35-encoded polypeptide chain release factor. The EMBO journal 15, 3127-3134

10. King, C. Y., Tittmann, P., Gross, H., Gebert, R., Aebi, M., and Wuthrich, K. (1997) Prioninducing domain 2-114 of yeast Sup35 protein transforms in vitro into amyloid-like filaments. Proc. Natl. Acad. Sci. U S A 94, 6618-6622

11. Paushkin, S. V., Kushnirov, V. V., Smirnov, V. N., and Ter-Avanesyan, M. D. (1997) In vitro propagation of the prion-like state of yeast Sup35 protein. Science 277, 381-383

12. Dobson, C. M. (2003) Protein folding and misfolding. Nature 426, 884-890

13. Dobson, C. M. (2004) Experimental investigation of protein folding and misfolding. Methods 34, 4-14

14. Dumoulin, M., and Dobson, C. M. (2004) Probing the origins, diagnosis and treatment of amyloid diseases using antibodies. Biochimie 86, 589-600

15. Steven, A., Baumeister, W., Johnson, L. N., and Perham, R. N. (2016) Molecular Biology of Assemblies and Machines, Garland Science

16. Makin, O. S., and Serpell, L. C. (2005) Structures for amyloid fibrils. FEBS J. 272, 59505961

17. Nielsen, L., Frokjaer, S., Brange, J., Uversky, V. N., and Fink, A. L. (2001) Probing the mechanism of insulin fibril formation with insulin mutants. Biochemistry 40, 8397-8409

18. Petkova, A. T., Leapman, R. D., Guo, Z., Yau, W.-M., Mattson, M. P., and Tycko, R. (2005) Self-propagating, molecular-level polymorphism in Alzheimer's B-amyloid fibrils. Science 307, 262-265

19. Kodali, R., Williams, A. D., Chemuru, S., and Wetzel, R. (2010) Abeta(1-40) forms five distinct amyloid structures whose beta-sheet contents and fibril stabilities are correlated. Journal of molecular biology 401, 503-517

20. Loksztejn, A., and Dzwolak, W. (2008) Chiral bifurcation in aggregating insulin: an induced circular dichroism study. J. Mol. Biol. 379, 9-16 
21. Lu, J. X., Qiang, W., Yau, W. M., Schwieters, C. D., Meredith, S. C., and Tycko, R. (2013) Molecular structure of beta-amyloid fibrils in Alzheimer's disease brain tissue. Cell 154, 1257 1268

22. Sulatskaya, A. I., Rodina, N. P., Kuznetsova, I. M., and Turoverov, K. K. (2017) Different conditions of fibrillogenesis cause polymorphysm of lysozyme amyloid fibrils. Journal of Molecular Structure 1140, $52-58$

23. Gordon, D. J., Balbach, J. J., Tycko, R., and Meredith, S. C. (2004) Increasing the amphiphilicity of an amyloidogenic peptide changes the $\beta$-sheet structure in the fibrils from antiparallel to parallel. Biophysical journal 86, 428-434

24. Mossuto, M. F., Dhulesia, A., Devlin, G., Frare, E., Kumita, J. R., de Laureto, P. P., Dumoulin, M., Fontana, A., Dobson, C. M., and Salvatella, X. (2010) The non-core regions of human lysozyme amyloid fibrils influence cytotoxicity. Journal of molecular biology 402, 783-796

25. Xue, W. F., Hellewell, A. L., Gosal, W. S., Homans, S. W., Hewitt, E. W., and Radford, S. E. (2009) Fibril fragmentation enhances amyloid cytotoxicity. The Journal of biological chemistry 284, 34272-34282

26. Korn, A., McLennan, S., Adler, J., Krueger, M., Surendran, D., Maiti, S., and Huster, D. (2018) Amyloid beta (1-40) toxicity depends on the molecular contact between phenylalanine 19 and leucine 34. ACS chemical neuroscience 9, 790-799

27. Tuite, M. F., Cox, B. S., and McLaughlin, C. S. (1987) A ribosome-associated inhibitor of in vitro nonsense suppression in [PSI] strains of yeast. FEBS letters 225, 205-208

28. Cox, B. S. (1964) $\left[\mathrm{PSI}^{+}\right]$, a cytoplasmic supresor of super-supressor in yeast. Genetics, $505-521$

29. Liebman, S., and Sherman, F. (1979) Extrachromosomal $\left[\mathrm{PSI}^{+}\right]$determinant suppresses nonsense mutations in yeast. Journal of bacteriology 139, 1068-1071

30. Tyedmers, J., Madariaga, M. L., and Lindquist, S. (2008) Prion switching in response to environmental stress. PLoS biology 6, e294

31. Chernova, T. A., Wilkinson, K. D., and Chernoff, Y. O. (2014) Physiological and environmental control of yeast prions. FEMS microbiology reviews 38, 326-344

32. Chernova, T. A., Romanyuk, A. V., Karpova, T. S., Shanks, J. R., Ali, M., Moffatt, N., Howie, R. L., O'Dell, A., McNally, J. G., and Liebman, S. W. (2011) Prion induction by the shortlived, stress-induced protein Lsb2 is regulated by ubiquitination and association with the actin cytoskeleton. Molecular cell 43, 242-252

33. Derkatch, I. L., Chernoff, Y. O., Kushnirov, V. V., Inge-Vechtomov, S. G., and Liebman, S. W. (1996) Genesis and variability of $\left[\mathrm{PSI}^{+}\right]$prion factors in Saccharomyces cerevisiae. Genetics 144, $1375-1386$ 
34. King, C.-Y., and Diaz-Avalos, R. (2004) Protein-only transmission of three yeast prion strains. Nature 428, 319

35. Diaz-Avalos, R., King, C.-Y., Wall, J., Simon, M., and Caspar, D. L. (2005) Strainspecific morphologies of yeast prion amyloid fibrils. Proc. Natl. Acad. Sci. U S A 102, 10165-10170

36. Ter-Avanesyan, M. D., Dagkesamanskaya, A. R., Kushnirov, V. V., and Smirnov, V. N. (1994) The SUP35 omnipotent suppressor gene is involved in the maintenance of the non-Mendelian determinant $\left[\mathrm{PSI}^{+}\right]$in the yeast Saccharomyces cerevisiae. Genetics 137, 671-676

37. Glover, J. R., Kowal, A. S., Schirmer, E. C., Patino, M. M., Liu, J. J., and Lindquist, S. (1997) Self-seeded fibers formed by Sup35, the protein determinant of $\left[P S I^{+}\right]$, a heritable prion-like factor of S. cerevisiae. Cell 89, 811-819

38. Toyama, B. H., Kelly, M. J., Gross, J. D., and Weissman, J. S. (2007) The structural basis of yeast prion strain variants. Nature 449, 233-237

39. Chang, H. Y., Lin, J. Y., Lee, H. C., Wang, H. L., and King, C. Y. (2008) Strain-specific sequences required for yeast $\left[\mathrm{PSI}^{+}\right]$prion propagation. Proc. Natl. Acad. Sci. U S A 105, 13345-13350

40. Kajava, A. V., Baxa, U., Wickner, R. B., and Steven, A. C. (2004) A model for Ure2p prion filaments and other amyloids: the parallel superpleated beta-structure. Proc. Natl. Acad. Sci. US A 101, 7885-7890

41. Shewmaker, F., Wickner, R. B., and Tycko, R. (2006) Amyloid of the prion domain of Sup35p has an in-register parallel beta-sheet structure. Proc. Natl. Acad. Sci. U S A 103, 1975419759

42. Shewmaker, F., Kryndushkin, D., Chen, B., Tycko, R., and Wickner, R. B. (2009) Two prion variants of Sup35p have in-register parallel beta-sheet structures, independent of hydration. Biochemistry 48, 5074-5082

43. Helsen, C. W., and Glover, J. R. (2012) Insight into molecular basis of curing of $\left[\mathrm{PSI}^{+}\right]$ prion by overexpression of 104-kDa heat shock protein (Hsp104). The Journal of biological chemistry 287, 542-556

44. Bondarev, S. A., Shchepachev, V. V., Kajava, A. V., and Zhouravleva, G. A. (2013) Effect of charged residues in the $\mathrm{N}$-domain of Sup35 protein on prion [PSI+] stability and propagation. The Journal of biological chemistry 288, 28503-28513

45. Bondarev, S. A., Shirokolobova, E. D., Trubitsina, N. P., and Zhouravleva, G. A. (2014) Modification of $\left[\mathrm{PSI}^{+}\right]$prion properties by the combination of amino acid changes within Sup35 protein N-domain. Mol biol (Mosk) 48, 314-321

46. Danilov, L. G., Matveenko, A. G., Ryzhkova, V. E., Belousov, M. V., Poleshchuk, O. I., Likholetova, D. V., Sokolov, P. A., Kasyanenko, N. A., Kajava, A. V., Zhouravleva, G. A., and Bondarev, S. A. (2019) Design of a New $\left[\mathrm{PSI}^{+}\right]$-No-More Mutation in SUP35 With Strong Inhibitory Effect on the $\left[\mathrm{PSI}^{+}\right]$Prion Propagation. Front Mol Neurosci 12, 274 
47. Vladimirov, Y. A., and Litvin, F. F. (1964) Photobiology and spectroscopic methods. Handbook of general biophisics $\mathbf{8}$

48. Fonin, A. V., Sulatskaya, A. I., Kuznetsova, I. M., and Turoverov, K. K. (2014) Fluorescence of dyes in solutions with high absorbance. Inner filter effect correction. PloS One 9, e103878

49. Greenfield, N. J. (2006) Using circular dichroism spectra to estimate protein secondary structure. Nature protocols 1, 2876-2890

50. Kjaergaard, M., Norholm, A. B., Hendus-Altenburger, R., Pedersen, S. F., Poulsen, F. M., and Kragelund, B. B. (2010) Temperature-dependent structural changes in intrinsically disordered proteins: formation of alpha-helices or loss of polyproline II? Protein science : a publication of the Protein Society 19, 1555-1564

51. Tinoco Jr, I., Bustamante, C., and Maestre, M. (1980) The optical activity of nucleic acids and their aggregates. Annual review of biophysics and bioengineering 9, 107-141

52. Naiki, H., Higuchi, K., Hosokawa, M., and Takeda, T. (1989) Fluorometric determination of amyloid fibrils in vitro using the fluorescent dye, thioflavin T1. Anal. Biochem. 177, 244-249

53. LeVine, H., 3rd. (1993) Thioflavine T interaction with synthetic Alzheimer's disease betaamyloid peptides: detection of amyloid aggregation in solution. Protein Sci. 2, 404-410

54. LeVine, H., 3rd. (1999) Quantification of beta-sheet amyloid fibril structures with thioflavin T. Methods Enzymol. 309, 274-284

55. Krebs, M. R., Bromley, E. H., and Donald, A. M. (2005) The binding of thioflavin-T to amyloid fibrils: localisation and implications. J. Struct. Biol. 149, 30-37

56. Wu, C., Biancalana, M., Koide, S., and Shea, J. E. (2009) Binding Modes of Thioflavin-T to the Single-Layer beta-Sheet of the Peptide Self-Assembly Mimics. J. Mol. Biol.

57. Biancalana, M., Makabe, K., Koide, A., and Koide, S. (2009) Molecular mechanism of thioflavin-T binding to the surface of beta-rich peptide self-assemblies. J. Mol. Biol. 385, 1052-1063

58. Biancalana, M., and Koide, S. (2010) Molecular mechanism of Thioflavin-T binding to amyloid fibrils. Biochimica et Biophysica Acta 1804, 1405-1412

59. Kuznetsova, I. M., Sulatskaya, A. I., Uversky, V. N., and Turoverov, K. K. (2012) Analyzing thioflavin $\mathrm{T}$ binding to amyloid fibrils by an equilibrium microdialysis-based technique. PloS One 7, e30724

60. Sulatskaya, A. I., Rodina, N. P., Polyakov, D. S., Sulatsky, M. I., Artamonova, T. O., Khodorkovskii, M. A., Shavlovsky, M. M., Kuznetsova, I. M., and Turoverov, K. K. (2018) Structural Features of Amyloid Fibrils Formed from the Full-Length and Truncated Forms of Beta-2Microglobulin Probed by Fluorescent Dye Thioflavin T. International journal of molecular sciences 19, E2762 
61. Sulatskaya, A. I., Kuznetsova, I. M., and Turoverov, K. K. (2011) Interaction of thioflavin $\mathrm{T}$ with amyloid fibrils: stoichiometry and affinity of dye binding, absorption spectra of bound dye. The journal of physical chemistry B 115, 11519-11524

62. Sulatskaya, A. I., Kuznetsova, I. M., Belousov, M. V., Bondarev, S. A., Zhouravleva, G. A., and Turoverov, K. K. (2016) Stoichiometry and Affinity of Thioflavin T Binding to Sup35p Amyloid Fibrils. PloS one 11, e0156314

63. Maskevich, A. A., Stsiapura, V. I., Kuzmitsky, V. A., Kuznetsova, I. M., Povarova, O. I., Uversky, V. N., and Turoverov, K. K. (2007) Spectral properties of thioflavin T in solvents with different dielectric properties and in a fibril-incorporated form. J. Proteome Res. 6, 1392-1401

64. Stsiapura, V. I., Kurhuzenkau, S. A., Kuzmitsky, V. A., Bouganov, O. V., and Tikhomirov, S. A. (2016) Solvent Polarity Effect on Nonradiative Decay Rate of Thioflavin T. The journal of physical chemistry A 120, 5481-5496

65. Ahmed, A. B., Znassi, N., Chateau, M. T., and Kajava, A. V. (2015) A structure-based approach to predict predisposition to amyloidosis. Alzheimer's \& dementia : the journal of the Alzheimer's Association 11, 681-690

66. Bondarev, S. A., Zhouravleva, G. A., Belousov, M. V., and Kajava, A. V. (2015) Structure-based view on $\left[\mathrm{PSI}^{+}\right]$prion properties. Prion 9, 190-199

67. Roche, D. B., Villain, E., and Kajava, A. V. (2017) Usage of a dataset of NMR resolved protein structures to test aggregation versus solubility prediction algorithms. Protein science: $a$ publication of the Protein Society 26, 1864-1869

68. Bondarev, S. A., Bondareva, O. V., Zhouravleva, G. A., and Kajava, A. V. (2018) BetaSerpentine: a bioinformatics tool for reconstruction of amyloid structures. Bioinformatics 34, 599-608

69. Derkatch, I. L., Bradley, M. E., and Liebman, S. W. (1998) Overexpression of the SUP45 gene encoding a Sup35p-binding protein inhibits the induction of the de novo appearance of the $\left[P S I^{+}\right]$prion. Proc. Natl. Acad. Sci. U S A 95, 2400-2405

70. Newnam, G. P., Wegrzyn, R. D., Lindquist, S. L., and Chernoff, Y. O. (1999) Antagonistic interactions between yeast chaperones Hsp104 and Hsp70 in prion curing. Molecular and cellular biology 19, 1325-1333

71. DePace, A. H., Santoso, A., Hillner, P., and Weissman, J. S. (1998) A critical role for amino-terminal glutamine/asparagine repeats in the formation and propagation of a yeast prion. Cell 93, $1241-1252$

72. Osherovich, L. Z., Cox, B. S., Tuite, M. F., and Weissman, J. S. (2004) Dissection and design of yeast prions. PLoS Biol 2, E86 
73. Shkundina, I. S., Kushnirov, V. V., Tuite, M. F., and Ter-Avanesyan, M. D. (2006) The role of the N-terminal oligopeptide repeats of the yeast Sup35 prion protein in propagation and transmission of prion variants. Genetics 172, 827-835

74. Dergalev, A. A., Alexandrov, A. I., Ivannikov, R. I., Ter-Avanesyan, M. D., and Kushnirov, V. V. (2019) Yeast Sup35 prion structure: two types, four parts, many variants. International journal of molecular sciences 20, E2633

75. Derkatch, I. L., Bradley, M. E., Zhou, P., and Liebman, S. W. (1999) The PNM2 mutation in the prion protein domain of SUP35 has distinct effects on different variants of the $\left[\mathrm{PSI}^{\dagger}\right]$ prion in yeast. Current Genetics 35, 59-67

76. Liu, J.-J., and Lindquist, S. (1999) Oligopeptide-repeat expansions modulate 'proteinonly'inheritance in yeast. Nature $\mathbf{4 0 0}, 573$

77. Allen, K. D., Wegrzyn, R. D., Chernova, T. A., Muller, S., Newnam, G. P., Winslett, P. A., Wittich, K. B., Wilkinson, K. D., and Chernoff, Y. O. (2005) Hsp70 chaperones as modulators of prion life cycle: novel effects of Ssa and Ssb on the Saccharomyces cerevisiae prion $\left[\mathrm{PSI}^{+}\right]$. Genetics 169, $1227-1242$

78. Studier, F. W., and Moffatt, B. A. (1986) Use of bacteriophage T7 RNA polymerase to direct selective high-level expression of cloned genes. Journal of molecular biology 189, 113-130

79. Serio, T. R., and Lindquist, S. L. (1999) $\left[P S I^{+}\right]$: an epigenetic modulator of translation termination efficiency. Annual review of cell and developmental biology 15, 661-703

80. Tanaka, M., Chien, P., Naber, N., Cooke, R., and Weissman, J. S. (2004) Conformational variations in an infectious protein determine prion strain differences. Nature 428, 323-328

81. Tanaka, M. (2010) A protein transformation protocol for introducing yeast prion particles into yeast. Methods in enzymology 470, 681-693

82. Matveenko, A. G., Drozdova, P. B., Belousov, M. V., Moskalenko, S. E., Bondarev, S. A., Barbitoff, Y. A., Nizhnikov, A. A., and Zhouravleva, G. A. (2016) SFPl-mediated prion-dependent lethality is caused by increased Sup35 aggregation and alleviated by Sis1. Genes to cells: devoted to molecular \& cellular mechanisms 21, 1290-1308

83. Sikorski, R. S., and Hieter, P. (1989) A system of shuttle vectors and yeast host strains designed for efficient manipulation of DNA in Saccharomyces cerevisiae. Genetics 122, 19-27

84. Kaiser, C., Michaelis, S., Mitchell, A., and Cold Spring Harbor Laboratory. (1994) Methods in yeast genetics: a Cold Spring Harbor Laboratory course manual, 1994 ed., Cold Spring Harbor Laboratory Press, Cold Spring Harbor, NY

85. Eaglestone, S. S., Ruddock, L. W., Cox, B. S., and Tuite, M. F. (2000) Guanidine hydrochloride blocks a critical step in the propagation of the prion-like determinant $\left[\mathrm{PSI}^{+}\right]$of Saccharomyces cerevisiae. Proc. Natl. Acad. Sci. U S A 97, 240-244 
86. Serio, T. R., Cashikar, A. G., Moslehi, J. J., Kowal, A. S., and Lindquist, S. L. (1999) Yeast prion $\left[\mathrm{PSI}^{+}\right]$and its determinant, Sup35p. Methods in enzymology 309, 649-673

87. Sulatskaya, A. I., Lavysh, A. V., Maskevich, A. A., Kuznetsova, I. M., and Turoverov, K. K. (2017) Thioflavin $\mathrm{T}$ fluoresces as excimer in highly concentrated aqueous solutions and as monomer being incorporated in amyloid fibrils. Scientific reports 7, 2146

88. O'Connor, D. V. P., D. (1984) Time-correlated Single Photon Counting. New-York, Academic Press, 37-54

89. Marquardt, D. W. (1963) An algorithm for least-squares estimation of non linear parameters. J. Soc. Ind. Appl. Math. 11, 431-441

90. Sanchez-Linares, I., Perez-Sanchez, H., Cecilia, J. M., and Garcia, J. M. (2012) HighThroughput parallel blind Virtual Screening using BINDSURF. BMC bioinformatics 13 Suppl 14, S13 


\section{Abbreviations}

$\left[P S I^{+}\right] \quad$ prion isoform of the Sup35p

Sup35NMp Sup35p fragment consisting of N-terminal and middle (M) region of the protein

R0 - R5 oligopeptide repeats of the amyloidogenic N-terminal domain of Sup35NMp

Sup35-M0 mutant form of Sup35NMp with the substitution in the R0 (Q33K/A34K)

Sup35-M1 mutant form of Sup35NMp with the substitution in the R1 (Y46K/Q47K)

Sup35-M2 mutant form of Sup35NMp with the substitution in the R2 (Q61K/Q62K)

Sup35-M3 mutant form of Sup35NMp with the substitution in the R3 (Q70K/Q71K)

Sup35-M4 mutant form of Sup35NMp with the substitution in the R4 (Q80K/Q81K)

Sup35-M5 mutant form of Sup35NMp with the substitution in the R5 (Q89K/Q90K)

ThT fluorescent probe thioflavin $\mathrm{T}$ 


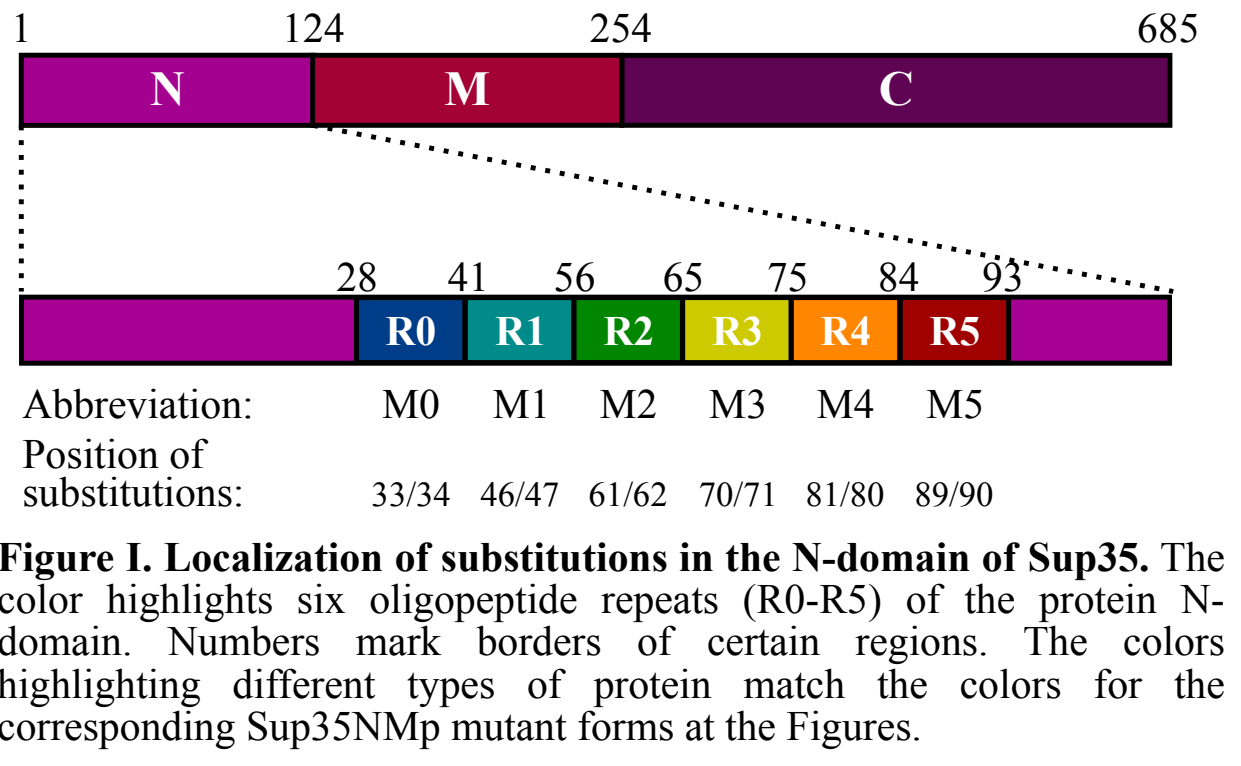




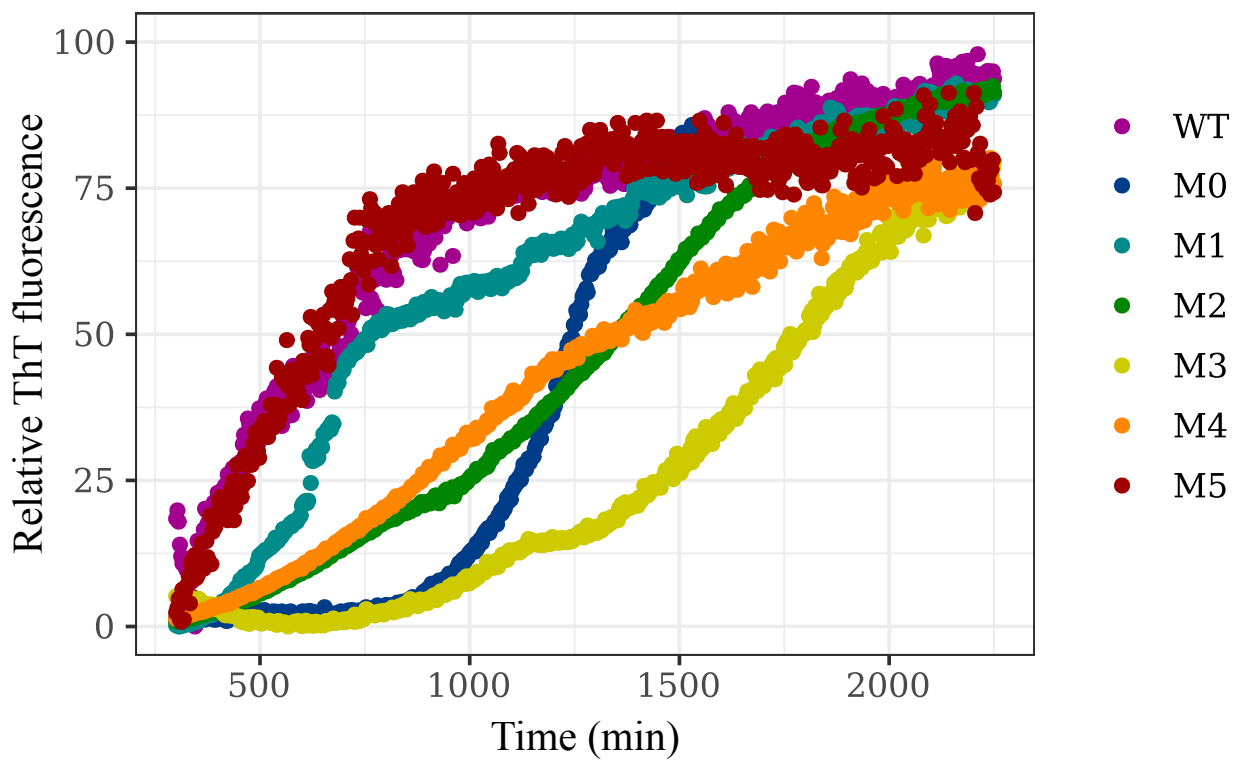

Figure II. Most of the analyzed substitutions affects the Sup35 aggregation. Results of ThT kinetic assay were presented. 
Table 2. Binding parameters of ThT to different types of Sup35NMp amyloid fibrils and characteristics of the bound dye.

\begin{tabular}{|c|c|c|c|c|c|}
\hline Object* & mode & $\begin{array}{c}\varepsilon_{i, \max } \mathbf{x} 10^{-4}, \\
\mathbf{M}^{-1} \mathbf{c m}^{-1}\end{array}$ & $\begin{array}{c}K_{b i} \mathbf{x 1 0}^{-5} \\
\mathbf{M}^{-1}\end{array}$ & $\mathbf{n}_{\mathbf{i}}$ & $\mathbf{q}_{\mathbf{i}}$ \\
\hline \multirow{2}{*}{$\begin{array}{l}\text { Sup35NMp } \\
\text { Wild type }\end{array}$} & 1 & $2.3 \pm 0.3$ & $70 \pm 10$ & $0.08 \pm 0.08$ & $0.35 \pm 0.04$ \\
\hline & 2 & $2.2 \pm 0.1$ & $0.21 \pm 0.07$ & $0.86 \pm 0.04$ & $0.15 \pm 0.02$ \\
\hline \multirow{2}{*}{$\begin{array}{c}\text { Sup35-M0 } \\
(\mathrm{Q} 33 \mathrm{~K} / \mathrm{A} 34 \mathrm{~K})\end{array}$} & 1 & $1.3 \pm 0.3$ & $71 \pm 12$ & $0.06 \pm 0.03$ & $0.25 \pm 0.03$ \\
\hline & 2 & $1.2 \pm 0.2$ & $0.19 \pm 0.03$ & $0.76 \pm 0.03$ & $0.04 \pm 0.02$ \\
\hline \multirow{2}{*}{$\begin{array}{c}\text { Sup35-M1 } \\
\text { (Y46K/Q47K) }\end{array}$} & 1 & $1.7 \pm 0.2$ & $71 \pm 10$ & $0.01 \pm 0.01$ & $0.32 \pm 0.05$ \\
\hline & 2 & $1.7 \pm 0.3$ & $0.23 \pm 0.04$ & $0.65 \pm 0.04$ & $0.03 \pm 0.02$ \\
\hline \multirow{2}{*}{$\begin{array}{c}\text { Sup35-M2 } \\
(\mathrm{Q} 61 \mathrm{~K} / \mathrm{Q} 62 \mathrm{~K})\end{array}$} & 1 & $3.0 \pm 0.3$ & $70 \pm 10$ & $0.02 \pm 0.07$ & $0.27 \pm 0.04$ \\
\hline & 2 & $1.6 \pm 0.2$ & $0.22 \pm 0.05$ & $0.80 \pm 0.04$ & $0.19 \pm 0.02$ \\
\hline \multirow{2}{*}{$\begin{array}{c}\text { Sup35-M3 } \\
(\mathrm{Q} 70 \mathrm{~K} / \mathrm{Q} 71 \mathrm{~K})\end{array}$} & 1 & $2.2 \pm 0.3$ & $70 \pm 11$ & $0.03 \pm 0.05$ & $0.22 \pm 0.06$ \\
\hline & 2 & $2.2 \pm 0.1$ & $0.22 \pm 0.02$ & $0.82 \pm 0.04$ & $0.15 \pm 0.02$ \\
\hline \multirow{2}{*}{$\begin{array}{c}\text { Sup35-M4 } \\
\text { (Q80K/Q81K) }\end{array}$} & 1 & $0.8 \pm 0.3$ & $70 \pm 12$ & $0.08 \pm 0.04$ & $0.21 \pm 0.04$ \\
\hline & 2 & $1.9 \pm 0.2$ & $0.20 \pm 0.04$ & $0.84 \pm 0.04$ & $0.16 \pm 0.02$ \\
\hline \multirow{2}{*}{$\begin{array}{c}\text { Sup35-M5 } \\
(\mathrm{Q89K} / \mathrm{Q} 9 \mathrm{~K})\end{array}$} & 1 & $1.7 \pm 0.3$ & $71 \pm 11$ & $0.08 \pm 0.03$ & $0.25 \pm 0.06$ \\
\hline & 2 & $1.9 \pm 0.3$ & $0.20 \pm 0.05$ & $0.86 \pm 0.05$ & $0.17 \pm 0.03$ \\
\hline
\end{tabular}

* The colors highlighting different types of protein match the colors for the corresponding Sup35NMp mutant forms at the Figures. 


\section{Figures}
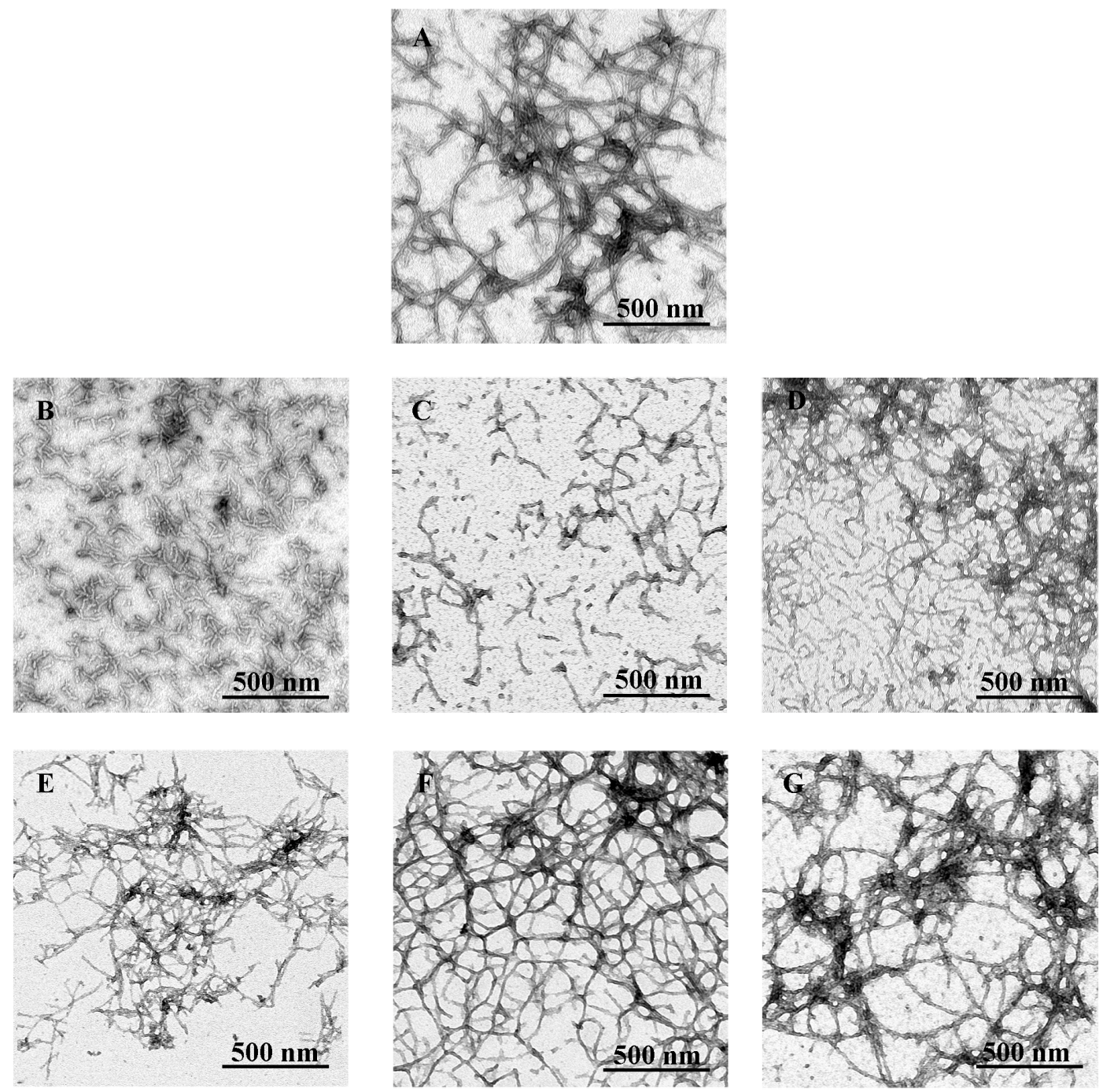

Figure 1. Images of amyloid fibrils formed from (A) wild type Sup35NMp and its mutant forms (B) Sup35-M0p, (C) Sup35-M1p, (D) Sup35-M2p, (E) Sup35-M3p, (F) Sup35-M4p and (G) Sup35-M5p, obtained using electron microscopy. The scale bar on the images is equal to $500 \mathrm{~nm}$. 

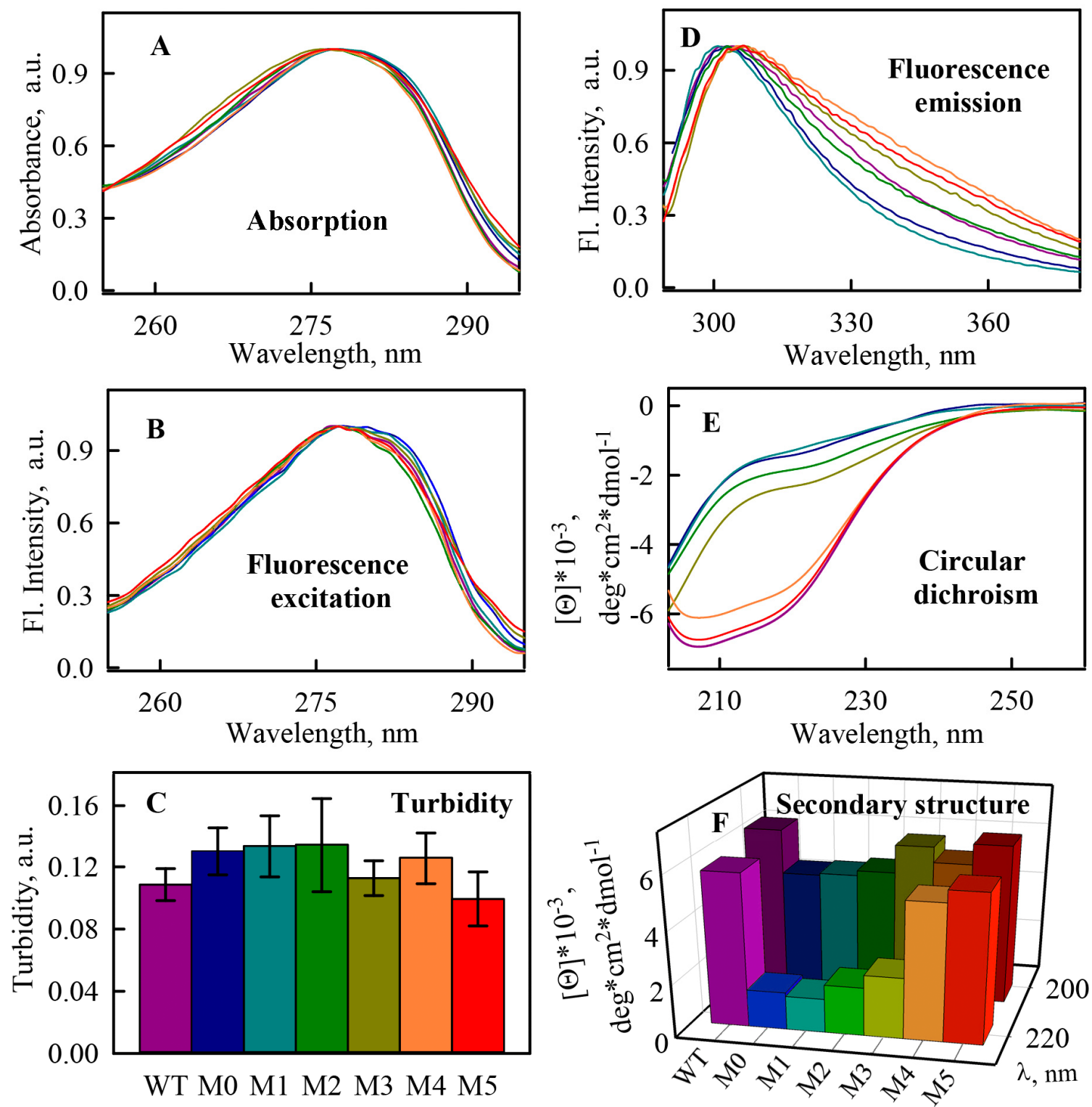

Figure 2. Photophysical properties of amyloid fibrils formed from Sup35NMp and its mutant forms. (A) Absorption, (B) fluorescence emission and (D) fluorescence excitation spectra, (C) turbidity, (E) CD spectra of the fibrils. (F) Absolute value of the molar ellipticity at the wavelengths 200 and $220 \mathrm{~nm}$ characterized the orderliness of the secondary structure of the samples. The results for the amyloid fibrils formed from different types of Sup35NMp are shown by the different colors: purple - Sup35NMp wild type (WT), blue - Sup35-M0p (M0), cyan - Sup35-M1p (M1), green - Sup35-M2p (M2), dark yellow - Sup35-M3p (M3), orange Sup35-M4p (M4), red - Sup35-M5p (M5). 

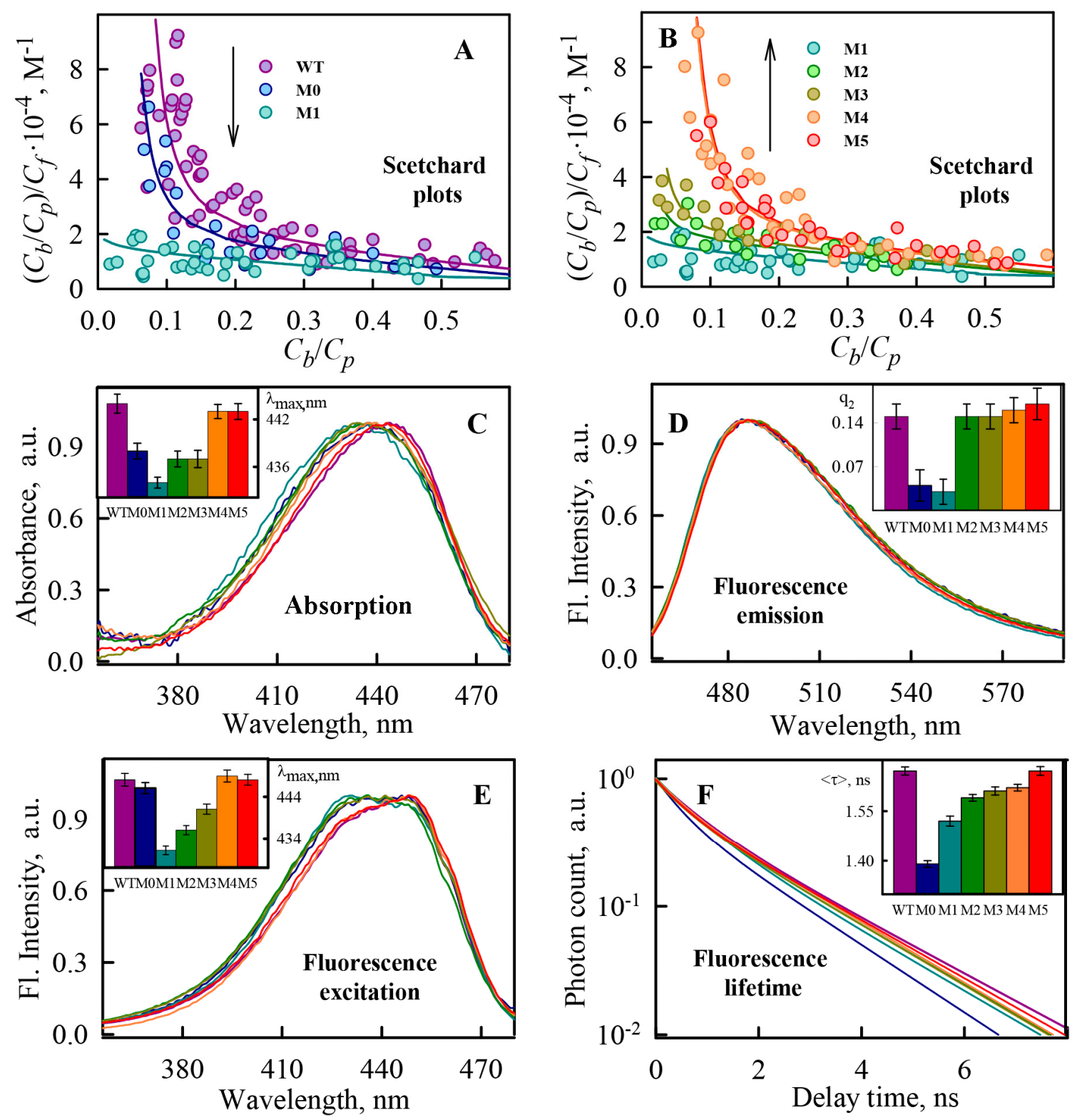

Figure 3. The interaction of Sup35NMp amyloid fibrils with a fluorescent probe thioflavin $\mathrm{T}$ (ThT). (A), (B) Scetchard plots for ThT binding to Sup35NMp amyloid fibrils. (C) Absorption, (D) fluorescence and (E) fluorescence excitation spectra and (F) fluorescence decay curves of the bound to fibrils ThT. At the (Inserts) to Panels $(\mathbf{C}),(\mathbf{E})$ the wavelengths of the ThT absorption and fluorescence spectra maximums, respectively, are shown. At the (Inserts) to Panels (D), (F) ThT fluorescence quantum yields (bound to the second fibrils mode) and lifetimes, respectively, are shown. Colors and designations are the same as at the Fig. 2. 
A

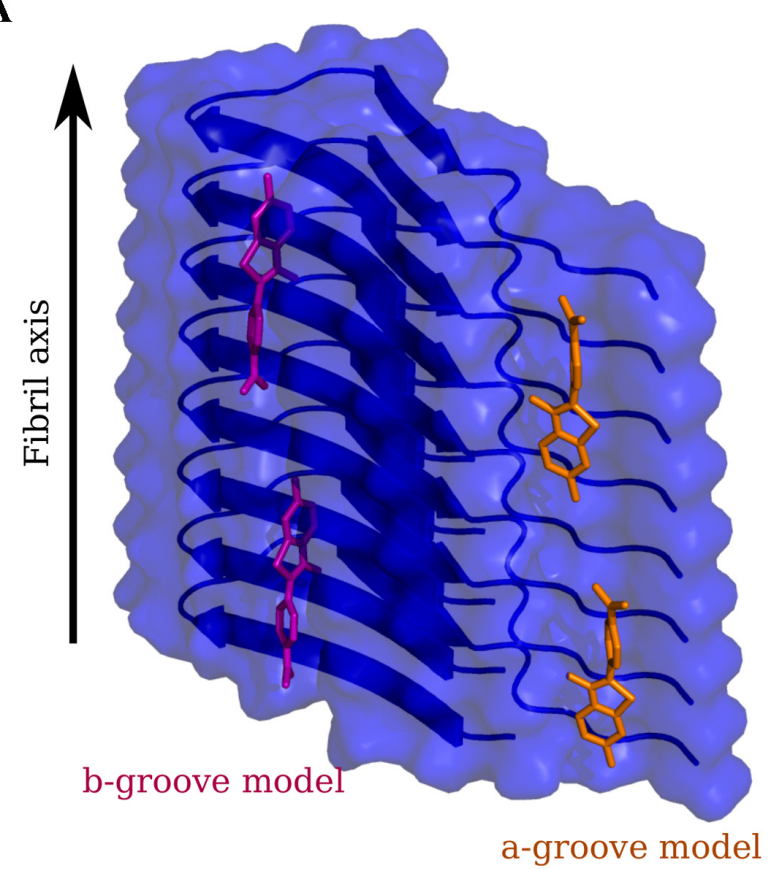

D

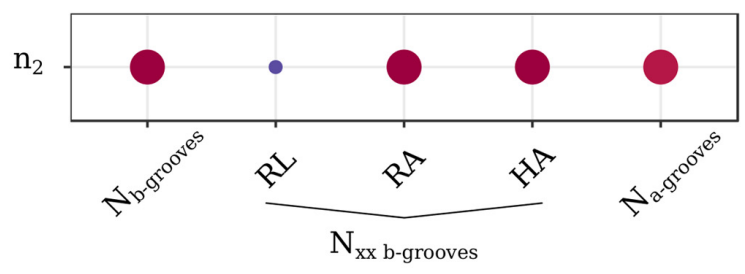

B

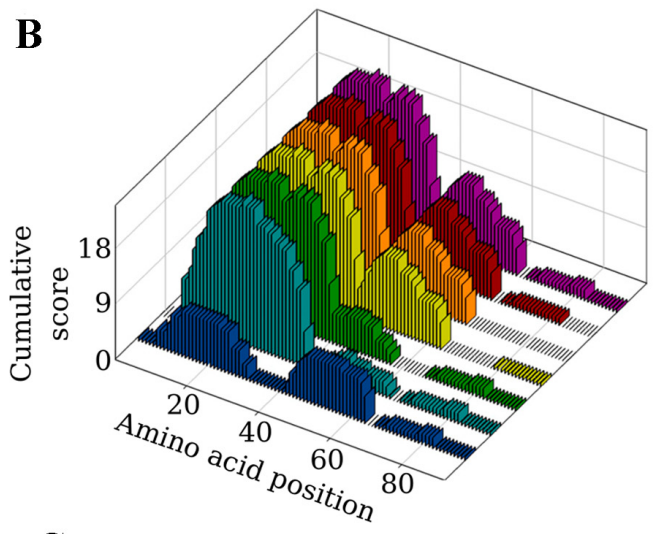

C

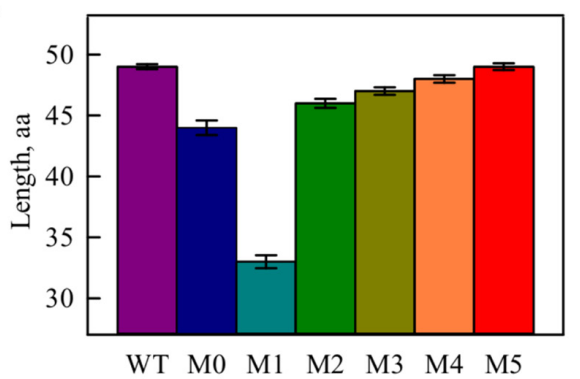

Spearman's correlation 0.20 .40 .60 .8 coefficient

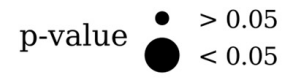

$\mathbf{E}$
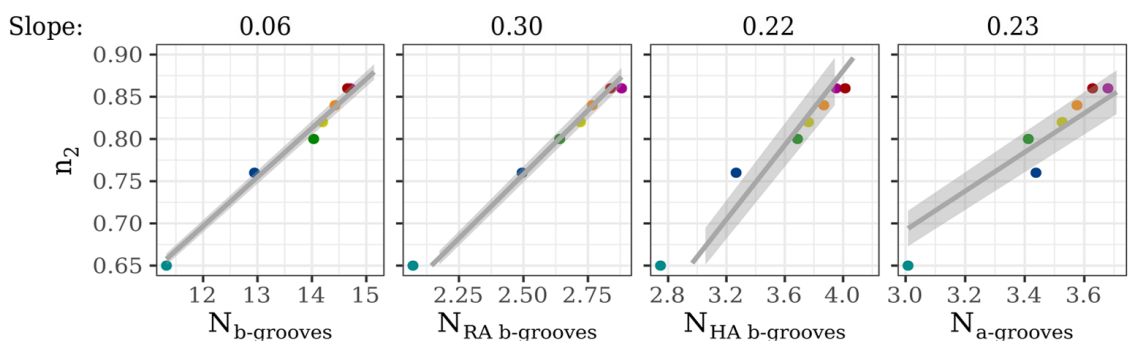

Figure 4. Correlation between numbers of predicted and experimentally determined ThT binding sites. (A) Two ThT binding models: incorporation of the into grooves formed by the side chains of amino acids, located in $\beta$-strands (b-groove model) or into the groove formed by the side chains and backbone at the base of the $\beta$-arch (a-groove model). The 2BEG PDB structure was used to illustrate these models, ThT molecules were docked to the fibril by the AutoDock Vina tool. (B) The frequency of inclusion of individual amino acid residues Sup35NMp in $\beta$-serpentine, calculated by the ArchCandy program. (C) Sup35-M0-M5p fragments of different lengths forming amyloid core. The predictions were made by the BetaSerpentine program. The median values are presented; error bars correspond to the confidential intervals. (D) The number of ThT binding sites for the second mode $\left(\mathrm{n}_{2}\right)$ correlates with predicted numbers of grooves $\left(\mathrm{N}_{\mathrm{b} \text {-rooves }}\right)$ and $\beta$-arches $\left(\mathrm{N}_{\mathrm{a} \text {-grooves }}\right)$. The Spearman's correlation coefficient was calculated for experimental data and the predicted number of ThT binding sites (mean value among all structures). We separately analyzed grooves comprising different types of amino acids: RL - one aRomatic $(\mathrm{F}, \mathrm{W}, \mathrm{Y})$ and one aLiphatic (A, L, M, I, V), RA - aromatic residue together with Any other, HA - groove with one Hydrophobic residue (F, W, Y, A, L, M, I, V) and Any other. Corresponding values were 
calculated based on structural arrangements of Sup35NMp provided by the BetaSerpentine program. An example was presented on the supplementary Figure S1. (E) Scatter plots demonstrating relationships for investigated parameters. Colors and designations are the same as at the Fig. 2. Lines correspond to linear model $\mathrm{y}=\mathrm{k} * \mathrm{x}$, gray area marks the $95 \%$ confidence interval for the model. 


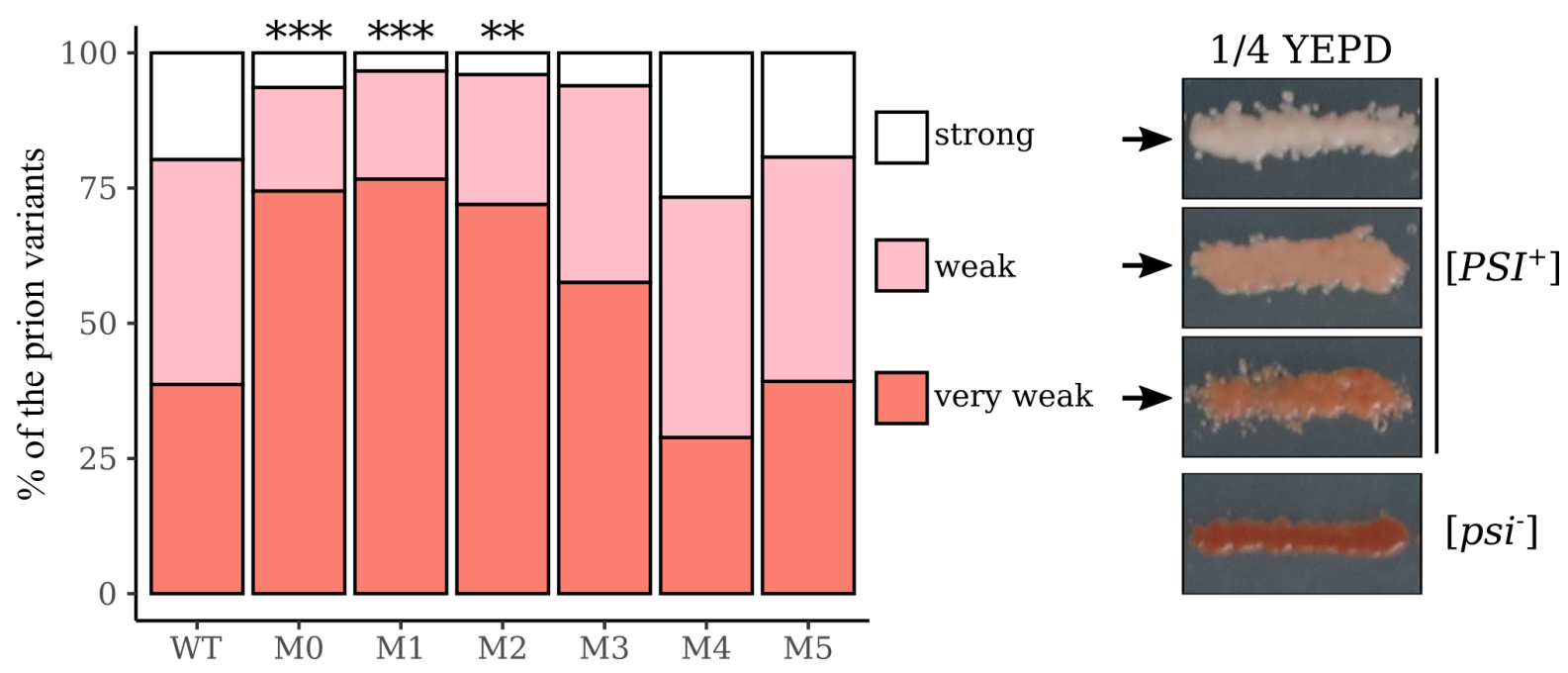

Figure 5. Charged residues in first oligopeptide repeats promote formation of the weak pron variants. The barplot illustrates ratios of different prion variants among all $\left[\mathrm{PSI}^{+}\right]$ transformants obtained after protein transformation. Phenotypes of corresponding yeast cells on the 1/4 YEPD are shown. ${ }^{* *} \ldots$ p-value $<0.01$ and $* * * \ldots$ p-value $<0.001$ according to Fisher's exact test. 


\begin{tabular}{|c|c|c|c|}
\hline & In silico & In vitro & In vivo \\
\hline $\begin{array}{l}\text { Sup35NMp } \\
\text { Wild type }\end{array}$ & 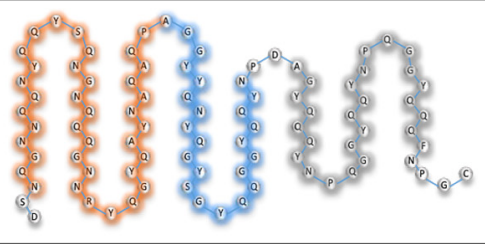 & $\begin{array}{l}\text {-high content of } \beta \text {-sheets }(>30 \%) \\
\text {-high fibrils length }(>1 \mu \mathrm{m}) \\
\text {-locally clumped fibrils } \\
\text {-ThT/protein stoichiometry } \sim 1 / 1.2 \\
\text { - high rigidity ThT binding sites } \\
\text { (q } \sim 0.15, \tau \sim 1.6 \mathrm{~ns})\end{array}$ & $\begin{array}{c}\text { prion } \\
\text { propagation }\end{array}$ \\
\hline $\begin{array}{c}\text { Sup35-M0 } \\
(\mathrm{Q} 33 \mathrm{~K} / \mathrm{A34K})\end{array}$ & 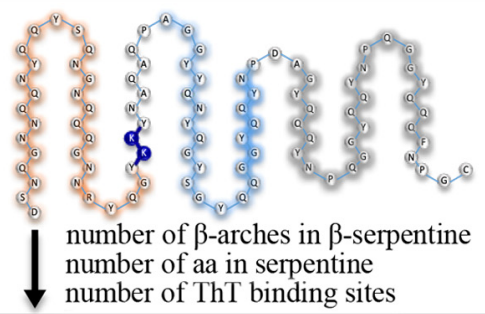 & $\begin{array}{l}\text { ordered structure ( } \beta \text {-sheets) } \\
\text { fibrils length } \\
\text { fibrils clumping } \\
\text { number of ThT binding sites } \\
\text { rigidity ThT binding sites } \\
\text { aggregation rate }\end{array}$ & $\begin{array}{c}\text { prion } \\
\text { loss }\end{array}$ \\
\hline $\begin{array}{c}\text { Sup35-M1 } \\
\text { (Y46K/Q47K) }\end{array}$ & $\begin{array}{l}\left.a_{0}\right) \\
\text { number of aa in } \beta \text {-serpentine } \\
\text { number of ThT binding sites }\end{array}$ & $\begin{array}{l}\text { ordered structure ( } \beta \text {-sheets) } \\
\text { fibrils length and diameter } \\
\text { fibrils clumping } \\
\text { number of ThT binding sites } \\
\text { rigidity ThT binding sites }\end{array}$ & $\begin{array}{l}\text { prion } \\
\text { loss }\end{array}$ \\
\hline $\begin{array}{c}\text { Sup35-M2 } \\
(\mathbf{Q 6 1 K / Q 6 2 K )})\end{array}$ & $\begin{array}{l}\text { number of } \beta \text {-arches in } \beta \text {-serpentine } \\
\text { number of aa in } \beta \text {-serpentine } \\
\text { number of ThT binding sites }\end{array}$ & $\begin{array}{l}\text { ordered structure ( } \beta \text {-sheets) } \\
\text { fibrils length } \\
\text { number of ThT binding sites } \\
\text { rigidity ThT binding sites } \\
\text { aggregation rate }\end{array}$ & $\begin{array}{c}\text { prion } \\
\text { loss }\end{array}$ \\
\hline $\begin{array}{c}\text { Sup35-M3 } \\
(\text { Q70K/Q71K) }\end{array}$ & $\begin{array}{l}\text { number of } \beta \text {-arches in } \beta \text {-serpentine } \\
\text { number of ThT binding sites }\end{array}$ & $\begin{array}{l}\text { ordered structure ( } \beta \text {-sheets) } \\
\text { fibrils diameter } \\
\text { aggregation rate } \\
\text { fibrils length } \\
\text { number of ThT binding sites } \\
\text { rigidity ThT binding sites }\end{array}$ & $\begin{array}{c}\text { prion } \\
\text { propagation }\end{array}$ \\
\hline $\begin{array}{c}\text { Sup35-M4 } \\
(\mathrm{Q} 80 \mathrm{~K} / \mathrm{Q} 81 \mathrm{~K})\end{array}$ & $\begin{array}{l}\underbrace{}_{0} \\
\text { number of } \beta \text {-arches in } \beta \text {-serpentine } \\
\text { number of a in } \beta \text {-serpentine }\end{array}$ & $\begin{array}{l}\text { aggregation rate } \\
\text { ordered structure ( } \beta \text {-sheets) } \\
\text { fibrils length } \\
\text { number of ThT binding sites }\end{array}$ & $\begin{array}{c}\text { prion } \\
\text { propagation }\end{array}$ \\
\hline $\begin{array}{c}\text { Sup35-M5 } \\
\text { (Q89K/Q90K) }\end{array}$ & 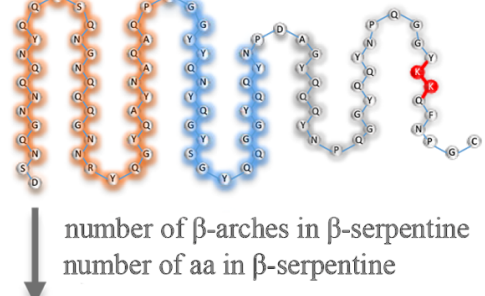 & $\begin{array}{l}\text { aggregation rate, } \\
\text { structure and ThT binding } \\
\text { are practically indentical } \\
\text { to that for wild type amyloids }\end{array}$ & $\begin{array}{c}\text { prion } \\
\text { propagation }\end{array}$ \\
\hline
\end{tabular}


Figure 6. The effect of mutations in the Sup35NMp amino acid sequence on the structural parameters, properties and characteristics of amyloid fibrils formed from this protein. The interrelation of the results obtained by in silico (parameters obtained using the program «BetaSerpentine»), in vitro and in vivo technics is presented. Colors and designations of mutations are the same as at the Fig. 2. In the scheme of the Sup35NMp amino acid sequence (proposed by Bondarev et al. (44)) shown the fragments forming $\beta$-serpentine, identified by the «ArchCandy» program, are highlighted using orange, blue and gray colors. The intensity of the fragments color for different fibrils characterizes the probability of the of an amino acid inclusion in $\beta$-serpentine. Near the arrows, the parameters and characteristics are shown, which values decreasing for fibrils with substitutions compared to that for wild-type Sup35NMp amyloid fibrils. The intensity of the arrow color indicates how much these characteristics have changed. 